\title{
Medication Review Service Implementation in Community Pharmacy Settings: Scoping Review with focus on Implementation Studies
}

\author{
Jaden Brandt*1, Mê-Linh Lề ${ }^{1,2}$, Sonja Jantscher ${ }^{3}$, Alě̌a Bricelj $^{4}$, Christopher Louizos ${ }^{1}$, \\ Sheila Ng, ${ }^{1}$ Kathryn Sibley ${ }^{5}$ \\ ${ }^{1}$ College of Pharmacy, Rady Faculty of Health Sciences, University of Manitoba, Winnipeg, Canada \\ ${ }^{2}$ Neil John Maclean Library, Rady Faculty of Health Sciences, University of Manitoba, Winnipeg, \\ Canada \\ ${ }^{3}$ Institute of Pharmacy, University of Graz, Austria \\ ${ }^{4}$ Faculty of Pharmacy, University of Ljubljana, Ljubljana, Slovenia \\ ${ }^{5}$ Knowledge Translation Platform, George and Faye Yee Centre for Healthcare Innovation, Department \\ of Community Health Sciences, Rady Faculty of Health Sciences, University of Manitoba, Winnipeg, \\ Canada \\ *Corresponding Author: umbrand2@,myumanitoba.ca / jkrbrandt@hotmail.com
}

Manuscript Word-Count (Excluding Abstract, Figures, Tables and References): 2,862 


\section{Medication Review Service Implementation in Community Pharmacy Settings: Scoping Review with focus on Implementation Studies}

Background: Medication reviews are a structured clinical intervention with the general goals of improving patient drug knowledge and detecting and resolving drug-related problems in an individual patient's medication regimen. A variety of barriers entrenched in the traditional drug distribution and dispensing model of pharmacy business has continued to challenge the implementation efforts of medication review services worldwide in the community pharmacy setting.

Main Objectives: i) Characterize original research studies that sought to enhance medication review service implementation in community pharmacy settings. ii) Categorize the broader corpus of scientific literature (beyond original implementation studies) on medication review service implementation in community pharmacy settings.

Methods: a broad systematic search strategy was applied to ovid MEDLINE, Embase, CINAHL and Cochrane Library to create an over-arching view and extensively ordered bibliography of the diverse research publication types dealing with the topic of medication review service implementation in the community pharmacy setting. A scoping review was subsequently conducted on original research studies that utilized various strategies to enhance the implementation of this service in community pharmacies. Data-charting evaluated the location of implementation studies, the strategies undertaken, the scale of implementation strategies, the use of DII (Dissemination, Implementation and Improvement) science theory, sample sizes, and DII outcomes.

Results: Of 5,947 records screened, 419 fulfilled the inclusion criteria (from abstract screening) to be deemed suitable for categorization and inclusion into the broader survey on this topic. Of these 419 publications, only 75 were original research specifically focused on enhancing the implementation of medication reviews in community pharmacy. A large majority of the publications were qualitative studies $(n=203)$. The remaining articles were improvement studies $(n=36)$, descriptive observational studies $(n=49)$, reviews $(n=69)$ and methodology papers $(n$ $=16$ ). Twenty-nine of these articles were deemed suitable for inclusion in more than one category. After full-text screening, 41 of the 75 implementation publications, representing 40 original studies, published between 1999-2019, were eligible for data-charting. The majority of these studies occurred in North America $(n=30)$, used some form of education as the most common implementation strategy $(\mathrm{n}=22)$ and measured 'adoption' (extent or frequency of medication reviews delivered) most frequently as an implementation outcome $(n=30)$. Just over half of the studies used a multi-faceted implementation strategy $(n=21)$. Only 9 studies used a theory, model or framework at any point in the research process to test hypotheses or explain empirical findings.

Conclusions: There is an abundance of publications addressing various issues surrounding medication review implementation in community pharmacies. However, the literature appears disproportionately represented by qualitative studies. There is also a need for more rigorously conducted implementation studies on medication review services in community pharmacy.

Key Words: Medication review, Implementation, Community pharmacy, Professional services 


\section{1) Background}

A medication review is a clinical patient-care service whereby a healthcare provider (usually a pharmacist) conducts a structured, critical evaluation of a patient's medication history, medication experience (i.e adherence, beliefs and preferences) and pharmacotherapy in the context of their disease states and health goals. ${ }^{1,2}$ Medication reviews thus aim to systematically detect and resolve various drug related problems or treatment related problems, with the collaboration and active involvement of the patient and prescriber, in order to optimize individual patient medication regimens for improved health outcomes. ${ }^{1,2}$ Evidence has supported the value of medication reviews to patient health and well-being by demonstrating increased medication adherence, improved control of chronic disease and reduction of drug-related problems. ${ }^{2-4}$

While the medication expertise and public accessibility of community pharmacists make them ideal candidates for delivering this important service, the traditional drug distribution and dispensing model of community pharmacy business has continued to challenge the implementation efforts of medication reviews and other clinical services worldwide in community pharmacy settings. ${ }^{5-7}$ Therefore, there is a need for a comprehensive characterization of the various strategies undertaken to implement medication reviews in the community pharmacy setting so as to overcome these barriers.

This scoping review, to our knowledge, is the first of its kind to systematically map, categorize and comment on the available literature relating to the broad topic of medication review implementation strategies in community pharmacy settings globally. Importantly, this review is meant to assist in informing other researchers in pharmacy practice to the current state of the broader literature on this topic prior to the conception of future implementation strategies. Another recent scoping review, focusing on patient and pharmacist perceptions as well as pharmacist conduct and outcomes of community pharmacist medication reviews in the United Kingdom, provides updated insight on issues affecting implementation. ${ }^{7}$ In contrast to that study, the primary objectives of this review were as follows:

i. $\quad$ Characterize original research studies that sought to enhance medication review service implementation in community pharmacy settings.

ii. Identify what proportion of the literature in this area is comprised of original implementation intervention studies compared to other types of investigation or article types (i.e reviews, qualitative studies etc.)

As the field of Dissemination, Implementation and Improvement (DII) science continues to develop and expand into sub-disciplines, including pharmacy, an additional focus was to assess the extent of influence from DII on this topic. ${ }^{8}$ This was formulated as a secondary objective:

iii. Identify to what extent the original implementation studies in this area applied tools, theories, frameworks and implementation relevant outcomes established according to the broader domain of DII science. 
This secondary objective was of interest for us to study because of the increasing reliance on theory, models or frameworks in planning studies or interpreting research findings in health service delivery. ${ }^{9}$ The use of theory, models or conceptual frameworks in DII is logically derived from principles of empiricism. Observed study results may either aid in the validation or refutation of a particular theory. Theories that have been well-established for their explanatory power can be further refined based upon more detailed research or used to test new hypotheses. It is for these reasons, in addition to the unique, product-focused (i.e retail) practice setting of community pharmacists, that the integration of DII principles can be viewed as essential for community pharmacy to make progress towards the expansion of clinical service provision.

\section{2) Method}

\section{1) Study Design}

This was a scoping review project that adhered to the corresponding extension of the PRISMA guidelines. ${ }^{10}$

\section{2) Protocol and Registration}

A protocol was previously registered with the Open Science Framework for this project. ${ }^{11}$

\section{3) Operational Definition of Medication Review}

There is significant inconsistency in the terminology used between countries to describe the pharmaceutical care process that is the 'medication review'. ${ }^{12-14}$ Because of this, we opted to use the consensus-based definition recently put forward by the Pharmaceutical Care Network of Europe $(\mathrm{PCNE})^{15}$ :

"Medication review is a structured evaluation of a patient's medicines with the aim of optimising medicines use and improving health outcomes. This entails detecting drug-related problems and recommending interventions"

We made note that this definition was inclusive of specific disease-based medication reviews and not only comprehensive medication reviews. Further, this definition makes allowance for the absence of patient interview as long as the process remains structured and relies on a patient medication history as the minimum required information source (though medication reviews almost always have a patient interview component). We also agreed with the PCNE in their assessment that medication reconciliation was not sufficient to constitute the definition of medication review. We also decided that services that presumably focus intentionally on a single newly prescribed medicine such as England's New Medicines Service (NMS) did not meet our definition of a medication review service. ${ }^{16}$

\section{4) Search Strategy}

A health sciences librarian, in collaboration with the research team, developed and tested the search strategy based on 20 pre-identified articles of relevance (available in supplemental file 1). The electronic search strategy for MEDLINE is available in supplemental file 1. The Ovid MEDLINE search also underwent peer-review following the PRESS checklist before the search 
was run in July 2018. ${ }^{17}$ Ovid MEDLINE, Embase, the Cochrane Library, and CINAHL were searched from inception to July 5, 2018 and restricted to English-only publications. A separate, broader search was also conducted with restriction to review articles to reduce the possibility that knowledge synthesis publications were not missed. There were no limits on the type of publication at the search execution stage. Lastly, searches of the journal Research in Social and Administrative Pharmacy were conducted because we felt this was the most relevant journal for the intersection of implementation science and the pharmacy profession.

\section{5) Screening Process and Eligibility Criteria}

The exported search results were uploaded to Rayyan; the online software we used for article screening. ${ }^{18}$ Articles were first independently title-screened by JB, SJ and AB (for nonreview articles) in a blinded fashion to eliminate any citations that were immediately discerned to be either irrelevant or clearly not matched to our research objectives (i.e improper indexing). The same process was done for review articles. There had to be at least 2 out of 3 votes on any given article to either exclude or include it into the next round of screening. Inter-rater agreement at this stage was $70 \%$ for the 572 review articles and $73 \%$ for the 5,375 non-review articles (i.e not proceeding to tie-breaker decision). Articles were then systematically excluded at the abstract/fulltext screening stage if the setting was not community pharmacy, if the research was incomplete or yet to be published (i.e protocol papers, conference abstracts), if the publication was strictly opinion, if the study was only focused on clinical, humanistic or economic outcomes of medication reviews or if there was no obvious focus on implementation of medication reviews. Articles were allowed to be excluded for multiple reasons if applicable.

\section{6) Article Categorization}

Once articles were deemed suitable for inclusion, they were labeled into 6 categories for future analysis; implementation intervention, improvement intervention, qualitative study, observational study, review (of any kind) or methodologic advancement (Table 2). These categories, partially decided in advance based on prior knowledge of the types of scientific publications and study types, evolved naturally throughout the screening process to more accurately convey the relevant content of the review.

\section{Table 1 here - Categories and Relevance of Literature Comprising Scoping Review (separate file}

2.7) Data Charting

\subsection{1) Process}

As per the primary objective, the data charting phase was focused solely on the original implementation intervention studies. Face validity of the data charting tool was ensured by having members of the research team review it prior to its use..$^{19}$ An early pilot exercise occurred between three members of the research team (JB, SJ, AB), which charted data from 20 different pre-selected 
papers. Concordance of results was deemed acceptable on all items ( $>80 \%$ agreement) with the highest agreement occurring on data items that were directly extractable and required minimal to no interpretation (i.e country of origin, intervention type, number of subjects etc..). Slight changes were made to the data charting templates after the pilot exercise; namely, removal of more ambiguous items such as 'key points', 'results' or 'main conclusions' as a measure to remove bias. JB independently data-charted all of the relevant original implementation studies and consulted on uncertainties in charting with members of the research team. Final data-charting results were confirmed by the research team.

\subsection{2) Data Items}

We charted basic study characteristics (author, year, geography) and factors relevant to the implementation study itself. As this was a scoping review, there was no focus on quality assessment of study design or methodology. The Effective Practice and Organization of Care (EPOC) taxonomy was used for categorizing distinct types of implementation interventions applicable to pharmacy practice. ${ }^{20}$ Slight deviations from the standard use of this taxonomy occurred only insofar as the ordering and classification of sub-divisions in the taxonomy. This was because strict adherence to the EPOC, as it was conceived, would have somewhat hindered the communication of our findings. Interventions were classed as either 'passive' or 'active' with the former being a change that occurred without necessarily changing the day-to-day operations of the community pharmacy (i.e financial incentive, national policy change or other interventions not directly enacted by pharmacy staff) and the latter focused on directly or indirectly enabling observable behaviour change in the workplace. We recorded whether these interventions were targeted directly at individuals, the pharmacy workplace or on a broader scale affecting the health system (i.e the entire pharmacy profession or the workplaces of multiple types of providers). The scoping review by Strifler et al., which catalogued the numerous theories, models, frameworks used in DII research, was used to check for use of these in the included studies. ${ }^{21}$ However, if other models, theories or frameworks were used beyond those listed by Strifler et al., they were not excluded from being reported. The conceptual taxonomy by Proctor et al. was used to differentiate the various implementation outcomes that the studies explicitly or implicitly used to evaluate the intervention effect. ${ }^{22}$

\section{3) Results}

\section{1) Broad Categorization}

Of the 419 identified articles of relevance, the largest category was qualitative studies (48.4\%); focusing on the perceptions of pharmacists, prescribers, patients, policy-makers, or other health providers on medication reviews in community pharmacies. The remaining categories in descending order of representation were original implementation studies $(17.9 \%)$, reviews (16.5\%), observational studies (11.7\%), improvement studies $(8.6 \%)$ and methodologic/service innovation (3.8\%). The categories were not completely mutually exclusive as 29 of the 419 articles $(6.4 \%)$ were deemed suitable for placement in two or more categories (i.e mixed-methods studies). 
Only 75 (18\%) of the 419 records that passed abstract screening comprised original projects that sought to improve implementation of medication reviews as they were defined. Furthermore, as this was the only category that proceeded to full-text screening, only 41 publications (covering 40 original studies) of these 75 truly fulfilled inclusion criteria suitable for consistent data-charting.

\section{Figure 1 here - Publication Selection Process (separate file)}

\section{2) Implementation Studies}

Of the 40 original implementation studies, 30 (75\%) were of North American origin (Canada or the United States), 8 (20\%) were from European countries and the remaining 2 (5\%) were from Australia. The publication rate of implementation studies on this topic has increased since the late 1990's, with 27 (67.5\%) of the studies being published in the 2010's. Thirty-six $(90 \%)$ of the studies provided sufficient information to categorize the PCNE 'type' of medication review; 1 was 'type 1' simple review (only medication history), 31 were 'type 2a' (patient interview + medication history), 1 was 'type 2b' (medication history + clinical information), and 3 studies implied some possibility of 'type 3' advanced review (medication history + patient interview + clinical information). Only $9(22 \%)$ studies reported on using a theoretical framework, model or tool in any stage of the research process to either plan the intervention or to explain or contextualize the findings. Two of the most recent studies used multiple theories or frameworks dependent upon the research phase. ${ }^{23,24}$

In terms of the operational scale of interventions, $9(22 \%)$ occurred at a regional or healthsystems level, 23 (56\%) occurred at the pharmacy level and $13(32 \%)$ occurred at the individual level ( 3 of which specifically focused solely on patients). Thirty-five (85\%) of the studies had 'active' implementation strategies, characterised by direct involvement on the part of the researcher(s) and/or collaboration from the participants, with the intention to improve medication review implementation. Most passive interventions were of a policy or financial inducement type.

Of the various implementation strategies employed, 19 studies were of only a single intervention category while the remainder were multi-faceted strategies; 12 having two intervention categories and 9 having three or more. By far the most common intervention category to improve medication review service implementation was education, delivered in various formats, occurring in $23(57 \%)$ of the studies.

The most frequent implementation outcome, using Proctor et al.'s taxonomy, was 'adoption', which was variably evaluated in 30 (75\%) studies. Only 24 (60\%) of the studies looked at more than one implementation outcome. Sustainability was only addressed or measured in two studies. 


\section{Table 2 here - Characteristics of Implementation Studies on Medication Review Services in} Community Pharmacy (separate file)

\section{4) Discussion}

There is a wealth of literature regarding implementation of medication reviews in community pharmacy settings. The various types of articles enumerated in our literature survey each offer distinct advantages for understanding the history and current state of medication review services in community pharmacy globally. However, an over-abundance of qualitative studies in this area may suggest the need for re-focusing research efforts away from opinions and perspectives on medication reviews and towards innovations in implementation and service improvement. Nevertheless, qualitative studies still invariably offer important insight on barriers and facilitators (often intangible social issues) that affect medication review services. Some review articles offer an important summary of many of these barriers and facilitators that should be considered by those developing implementation strategies for different community pharmacy services. ${ }^{65-67}$ Pharmacists' attitudes and attributes, relationships with other healthcare professionals and workplace environment (i.e staffing levels, consultation area, information resources) have all been identified as factors which may either impede or facilitate the implementation of medication review services.

In regards to the targeted focus of implementation studies in this project, a number of observations bear mention. There is clearly a need for further implementation studies occurring outside of North America. This is particularly true for countries in Latin America, South America, Africa and Asia. Though, the absence of studies in these areas may be indicative of unique limitations facing the pharmacy profession in those regions, such as human resource shortages, which may supersede the delivery of medication review services as a priority focus. ${ }^{68,69}$ Additionally, the exclusion of non-English language articles may have biased us slightly towards this observation.

Educational interventions have traditionally been the strategy of choice in implementation studies in this area. ${ }^{55,56,59-64}$ This finding is in accordance with other observations of applied DII strategies for evidence-based healthcare. ${ }^{70}$ Additional strategies such as practice support (i.e professional communities, organizational culture, role-expansion, clinical teamwork) and workplace support (i.e workplace restructuring, human resource changes, technology) may be better received by pharmacists compared to policy or finance based interventions that are enacted broadly without sufficient consultation or preparation from community pharmacy businesses. ${ }^{71}$ Contrary to expectations, the use of a multi-faceted strategy, while perhaps more acceptable or appealing in many cases, is not necessarily more effective on average than single intervention implementation strategies based on the broader DII literature. ${ }^{72}$

The observed focus on 'adoption' as the most common implementation outcome measure in the data-charted studies, at the frequent exclusion of other conceptual implementation outcomes, will necessarily miss important contextual information that would otherwise assist DII researchers in pharmacy. For example, 'adoption', in the studies that were data-charted, was most often measured as a change in frequency or quantity of medication reviews performed. ${ }^{25-27,31,33,35-37,40}$ 
A singular focus on the number of services rendered over a study period does not provide information about a particular implementation strategy's favourability among the pharmacy staff (i.e acceptability), the ethical reception by the pharmacy, collaborative healthcare providers and patients (i.e appropriateness), the ease by which it is employed (i.e feasibility), to what extent the intervention was adhered to as described (i.e fidelity), or the likelihood of it's being maintained into the future (i.e sustainability). This review reveals that greater emphasis on other implementation outcomes should occur. Recent studies such as Garcia-Cardenas et al. and Lelubre et al. which evaluate multiple implementation outcomes, and use DII theoretical frameworks or models, are steps in the right direction for advancing DII science in community pharmacy practice. ${ }^{23,24}$ Newer DII frameworks specific to pharmacy practice may also be adopted for research use. ${ }^{73,74}$ Nonetheless, careful selection of theory should be based on various factors specific to the research context and study setting. Guidance in this regard is provided elsewhere to assist pharmacy researchers new to DII science. ${ }^{9,75,76}$

Lastly, superior DII study methodology such as cluster randomized controlled trials or stepped-wedge designs, while more difficult and costly to conduct, may provide further insight into which implementation strategies or component interventions are most promising for improving service implementation in the community pharmacy setting. ${ }^{77,78}$

\section{1) Strengths and Limitations}

This study had a number of important methodological strengths. Utilization of a broad, systematic search strategy followed by a rigorous article screening process ensured highprobability of capturing the majority of the relevant literature. Data-charting accuracy and validity was also enhanced by use of previously established categorization schemas, explicit definitions and research team review. Despite these strengths, a few important limitations should be considered when interpreting our findings. First, articles in categories other than implementation studies were not full-text screened, making it likely that there was some degree of publication miscategorization in the available bibliography (supplemental file 2), though we do not anticipate this to undermine our general findings. Second, we did not report on the success or failure of the specific implementation studies in our data-charting activity. This was because the precise definitions of the measures used, the intervention strategies employed and the circumstances surrounding each study (i.e the regulatory environment, workplace settings etc.) were too variable for us to be comforted in reporting any final conclusions in the absence of their accompanying context. As DII is a "real-world" science, controlling for variability between studies is difficult because of contextual differences between study settings and implementation strategies. ${ }^{79}$ This problem is compounded in the case of complex interventions such as medication reviews, which themselves may differ in quality and delivery expectations dependent on context (information access, care-planning, communication requirements etc.), further hindering or aiding implementation efforts. ${ }^{80}$ Thirdly, our project focused solely on medication reviews conducted in community pharmacy settings and so other medication reviews involving pharmacists, such as those of Australia's Home Medicines Review program, were excluded as these other service settings may encompass different barriers and facilitators for implementation. ${ }^{81}$ 


\section{5) Conclusions}

In spite of a voluminous literature on pharmacist medication reviews in community pharmacy, there appears to be an over-abundance of qualitative study publications relative to other types of original research. However, a high-level knowledge synthesis of these studies would be needed to determine the extent to which the findings are replicable or to confirm over-saturation of research findings in this area.

There is a need for more rigorously conducted implementation studies on medication review services in community pharmacy. Future implementation studies should consider using DII theories, frameworks or models and report on more implementation outcomes than has been done previously. Implementation strategies should be justified based on both the local context and readings of the DII literature.

\section{Acknowledgements}

The authors are grateful to the three reviewers for their helpful suggestions to improve the manuscript quality.

\section{Funding Source}

There was no source of external funding that supported this work.

\section{Declaration of Interests}

The authors declare no conflicts of interest as it relates to this work.

\section{References}

1. De Oliveira Santos Silva R, Macêdo LA, Dos Santos GA, Aguiar PM, De Lyra DP. Pharmacistparticipated medication review in different practice settings: Service or intervention? An overview of systematic reviews. PLoS One. 2019;14(1):1-24. doi:10.1371/journal.pone.0210312

2. Jokanovic N, Tan EC, Sudhakaran S, et al. Pharmacist-led medication review in community settings: An overview of systematic reviews. Res Social Adm Pharm. 2017;13(4):661-685.

3. Kallio SE, Kiiski A, Airaksinen MSA, et al. Community Pharmacists' Contribution to Medication Reviews for Older Adults: A Systematic Review. J Am Geriatr Soc. 2018.

4. Buss VH, Shield A, Kosari S, Naunton M. The impact of clinical services provided by community pharmacies on the Australian healthcare system : a review of the literature. 2018:1-10.

5. Latif A. Community pharmacy Medicines Use Review : current challenges. Integr Pharm Res Pract. 2018;(7):83-92.

6. Brown D. The paradox of pharmacy: A profession's house divided. J Am Pharm Assoc. 2012;52(6):e139-e143. doi:10.1331/JAPHA.2012.11275

7. Stewart D, Whittlesea C, Dhital R, Newbould L, McCambridge J. Community pharmacist led medication reviews in the UK: A scoping review of the medicines use review and the new medicine service literatures. Res Social Adm Pharm. April 2019.

doi:10.1016/j.sapharm.2019.04.010 
8. Curran GM, Shoemaker SJ. Advancing pharmacy practice through implementation science. Res Soc Adm Pharm. 2017;13(5):889-891. doi:10.1016/j.sapharm.2017.05.018

9. Nilsen P. Making sense of implementation theories, models and frameworks. Implement Sci. 2015;10(53):1-13. doi:10.1186/s13012-015-0242-0

10. Tricco AC, Lillie E, Zarin W, et al. PRISMA Extension for Scoping Reviews ( PRISMA-ScR ): Checklist and Explanation. 2018. doi:10.7326/M18-0850

11. Brandt J, Sibley K, Lê M-L, Louizos C. Implementation of Medication Review Services in Community Pharmacy Settings: Scoping Review Protocol. doi:10.17605/OSF.IO/KW9JF

12. American Pharmacists Association. Medication therapy management in pharmacy practice: core elements of an MTM service model (version 2.0). J Am Pharm Assoc (2003). 2008;48(3):341-353.

13. Bassi M, Wood K. Medicines use reviews: Time for a new name? Int J Pharm Pract. 2009;17:B4B5.

14. Dolovich L, Consiglio G, MacKeigan L, et al. Uptake of the MedsCheck annual medication review service in Ontario community pharmacies between 2007 and 2013. Can Pharm J (Ott). 2016;149(5):293-302.

15. Griese-Mammen N, Hersberger KE, Messerli M, et al. PCNE definition of medication review: reaching agreement. Int J Clin Pharm. 2018;40(5):1199-1208. doi:10.1007/s11096-018-0696-7

16. Latif A, Watmough D, Salema NE, Elliott RA, Boyd MJ, Waring J. The New Medicines Service (NMS): Understanding its delivery in everyday practice. Int J Pharm Pract. 2014;22:67.

17. McGowan J, Sampson M, Salzwedel DM, Cogo E, Foerster V, Lefebvre C. PRESS Peer Review of Electronic Search Strategies: 2015 Guideline Statement. J Clin Epidemiol. 2016;75:40-46. doi:10.1016/j.jclinepi.2016.01.021

18. Ouzzani M. Rayyan — a web and mobile app for systematic reviews. Syst Rev. 2017;(2016):1-10. doi:10.1186/s13643-016-0384-4

19. Salkind N. Face Validity. In: Encyclopedia of Research Design. 2455 Teller Road, Thousand Oaks California 91320 United States: SAGE Publications, Inc.; 2010. doi: $10.4135 / 9781412961288$

20. Effective Practice and Organisation of Care (EPOC). The EPOC Taxonomy of Health Systems Interventions. Vol 1.; 2016.

21. Strifler L, Cardoso R, McGowan J, et al. Scoping review identifies significant number of knowledge translation theories, models, and frameworks with limited use. J Clin Epidemiol. 2018. doi:10.1016/j.jclinepi.2018.04.008

22. Proctor E, Silmere H, Raghavan R, et al. Outcomes for implementation research: Conceptual distinctions, measurement challenges, and research agenda. Adm Policy Ment Heal Ment Heal Serv Res. 2011;38(2):65-76. doi:10.1007/s10488-010-0319-7

23. Lelubre M, Wuyts J, Maesschalck J, et al. Implementation study of an intermediate medication review in Belgian community pharmacies. Res Soc Adm Pharm. 2019;(In Press):1-14. doi:10.1016/j.sapharm.2018.09.002

24. Garcia-Cardenas V, Benrimoj SI, Ocampo CC, Goyenechea E, Martinez-Martinez F, Gastelurrutia MA. Evaluation of the implementation process and outcomes of a professional pharmacy service in a community pharmacy setting. A case report. Res Social Adm Pharm. 2017;13(3):614-627. 
25. Murray ME, Barner JC, Pope ND, Comfort MD. Impact and Feasibility of Implementing a Systematic Approach for Medication Therapy Management in the Community Pharmacy Setting: A Pilot Study. J Pharm Pract. 2018:897190018779847.

26. Grootendorst P, Shim M, Tieu J. Uptake and impact of regulated pharmacy technicians in Ontario community pharmacies. Can Pharm J (Ott). 2018;151(3):197-202.

27. Lengel M, Kuhn CH, Worley M, Wehr AM, McAuley JW. Pharmacy technician involvement in community pharmacy medication therapy management. J Am Pharm Assoc (2003). 2018;58(2):179-185.e172.

28. Mailloux L, Yates S, Lessig K, et al. Changing patient perceptions of medication therapy management: What method of education is most effective? J Am Pharm Assoc. 2017;57(3).

29. Garcia-Cardenas V, Perez-Escamilla B, Fernandez-Llimos F, Benrimoj SI. The complexity of implementation factors in professional pharmacy services. Res Soc Adm Pharm. 2017:11-13. doi:10.1016/j.sapharm.2017.05.016

30. Isetts B. Integrating Medication Therapy Management (MTM) Services Provided by Community Pharmacists into a Community-Based Accountable Care Organization (ACO). Pharm (Basel, Switzerland). 2017;5(4).

31. Houle SKD, Charrois TL, Faruquee CF, Tsuyuki RT, Rosenthal MM. A randomized controlled study of practice facilitation to improve the provision of medication management services in Alberta community pharmacies. Res Social Adm Pharm. 2017;13(2):339-348.

32. Tomaszewski D, Cernohous T, Vaidyanathan R. Evaluating Patient Preferences for Different Incentive Programs to Optimize Pharmacist-Provided Patient Care Program Enrollment. J Manag care Spec Pharm. 2017;23(11):1140-1147.

33. Stafford R, Thomas J, Payakachat N, et al. Using an array of implementation strategies to improve success rates of pharmacist-initiated medication therapy management services in community pharmacies. Res Social Adm Pharm. 2017;13(5):938-946.

34. Smith MG, Ferreri SP, Brown P, Wines K, Shea CM, Pfeiffenberger TM. Implementing an integrated care management program in community pharmacies: A focus on medication management services. $J$ Am Pharm Assoc (2003). 2017;57(2):229-235.e221.

35. Vande Griend JP, Rodgers M, Nuffer W. Effect of an Advanced Pharmacy Practice Experience on Medication Therapy Management Services in a Centralized Retail Pharmacy Program. J Manag care Spec Pharm. 2017;23(5):561-565.

36. Pagano GM, Groves BK, Kuhn CH, Porter K, Mehta BH. A structured patient identification model for medication therapy management services in a community pharmacy. $J$ Am Pharm Assoc. 2017;57(5):619-623.e1. doi:10.1016/j.japh.2017.05.010

37. Schoenrock DL, Hartkopf K, Boeckelman C. Development and implementation of a pharmacistrun comprehensive medication review program in Wisconsin. Am J Health Syst Pharm. 2016;73(23):S155-S160.

38. Bradley CL, Luder HR, Beck AF, et al. Pediatric asthma medication therapy management through community pharmacy and primary care collaboration. J Am Pharm Assoc (2003). 2016;56(4):455460 . 
39. Huet AL, Frail CK, Lake LM, Snyder ME. Impact of passive and active promotional strategies on patient acceptance of medication therapy management services. J Am Pharm Assoc (2003). 2015;55(2):178-181.

40. Lasota ST, McConaha JL, Lynch KJ, Greco AJ. Implementation strategy to bring medication therapy management into high-volume community pharmacies. Am J Pharm Benefits. 2015;7(2):92-99.

41. Lester CA, Helmke JL, Kaefer TN, et al. Integrating Components of Medication Therapy Management Services into Community Pharmacy Workflow. 2014;5(2).

42. Grindrod K, Sanghera N, Rahmaan I, Roy M, Tritt M. Living MedsCheck: Learning how to deliver MedsCheck in community practice in Ontario. Can Pharm J (Ott). 2013;146(1):33-38.

43. Teichert M, Luijben SN, Wereldsma A, et al. Implementation of medication reviews in community pharmacies and their effect on potentially inappropriate drug use in elderly patients. Int J Clin Pharm. 2013;35(5):719-726.

44. Riley K. Enhanced medication management services in the community: A win-win proposal from an economic, clinical and humanistic perspective. Can Pharm J. 2013;146(3):162-168.

45. Pattin A, Szyskowski J. The development of a patient care center in a supermarket chain pharmacy. J Pharm Pract. 2013;26(1):32-35.

46. Houle SKD, Chuck AW, Tsuyuki RT. Blood pressure kiosks for medication therapy management programs: business opportunity for pharmacists. J Am Pharm Assoc (2003). 2012;52(2):188-194.

47. Lam A, Odegard PS, Gardner J. School of pharmacy-based medication therapy management program: development and initial experience. J Am Pharm Assoc (2003). 2012;52(3):398-404.

48. Ross LA, Bloodworth LS. Patient-centered health care using pharmacist-delivered medication therapy management in rural Mississippi. J Am Pharm Assoc (2003). 2012;52(6):802-809.

49. Schuh MJ, Crumb DJ, Dubois J. Building a pharmacist-managed pharmacotherapy medication therapy management practice. Consult Pharm. 2011;26(6):404-413.

50. Dualde E, Faus MJ, Santonja FJ, Fernandez-Llimos F. Effectiveness of a videoconference training course on implementing pharmacy services. Pharm World Sci. 2009;31(6):638-642.

51. Trapskin K, Johnson C, Cory P, Sorum S, Decker C. Forging a novel provider and payer partnership in Wisconsin to compensate pharmacists for quality-driven pharmacy and medication therapy management services. J Am Pharm Assoc (2003). 2009;49(5):642-651.

52. Lewis NJW, Bugdalski-Stutrud C, Abate MA, Blommel M, Wu CH, Gaither CA. The Medication Assessment Program: comprehensive medication assessments for persons taking multiple medications for chronic diseases. J Am Pharm Assoc (2003). 2008;48(2):171-180.

53. Blenkinsopp A, Celino G, Bond C, Inch J. Medicines use reviews: The first year of a new community pharmacy service. Pharm J. 2007;278(7440):218-223.

54. Blenkinsopp A, Bond C, Celino G, Inch J, Gray N. Medicines Use Review: adoption and spread of a service innovation. Int J Pharm Pract. 2008;16(4):271-276. doi:10.1211/ijpp.16.4.0010

55. Rouleau R, Beauchesne MF, Laurier C. Impact of a continuing education program on community pharmacists' interventions and asthma medication use: a pilot study. Ann Pharmacother. 2007;41(4):574-580. 
56. Jaffray M, Krska J, Lee AJ, Bond CM. The MEDMAN project: Evaluation of the medicines management training for community pharmacists. Pharm Educ. 2007;7(3):207-214.

57. Pai AK. Integration of a clinical community pharmacist position: emphasis on workflow design. $J$ Am Pharm Assoc (2003). 2005;45(3):400-403.

58. Stuurman-Bieze AGG, de Boer WO, Kokenberg MEAP, Hugtenburg JG, de Jong-van den Berg LTW, Tromp TFJ. Complex pharmaceutical care intervention in pulmonary care: part A. The process and pharmacists' professional satisfaction. Pharm World Sci. 2005;27(5):376-384.

59. Aston J, Black P. Does participation in formal postgraduate studies have a positive impact on pharmacists' professional activities? Pharm J. 2006;276(7387):175-179.

60. Lopatka H, Bachynsky J. Evaluation of a community pharmacy reimbursement model for cognitive services-experiment and survey. J Pharm Financ Econ Policy. 2004;13(1):69-94.

61. Carter BL, Chrischilles EA, Scholz D, Hayase N, Bell N. Extent of services provided by pharmacists in the Iowa Medicaid Pharmaceutical Case Management program. J Am Pharm Assoc (Wash). 2003;43(1):24-33.

62. March G, Gilbert A, Roughead E, Quintrell N. Developing and evaluating a model for pharmaceutical care in Australian community pharmacies. Int J Pharm Pract. 1999;7(4):220-229.

63. Barner JC, Bennett RW. Pharmaceutical care certificate program: assessment of pharmacists' implementation into practice. J Am Pharm Assoc (Wash). 1999;39(3):362-367.

64. Chen TF, Crampton M, Krass I, Benrimoj SI. Collaboration between community pharmacists and GPs - The medication review process. J Soc Adm Pharm. 1999;16(3):145-156.

65. Luetsch K. Attitudes and attributes of pharmacists in relation to practice change - A scoping review and discussion. Res Soc Adm Pharm. 2017;13(3):440-455.e11. doi:10.1016/j.sapharm.2016.06.010

66. Luetsch K. Pharmacists' personality traits-barriers to practice change? Int J Pharm Pract. 2016;24:19.

67. Hossain LN, Fernandez-Llimos F, Luckett T, et al. Qualitative meta-synthesis of barriers and facilitators that influence the implementation of community pharmacy services: perspectives of patients, nurses and general medical practitioners. BMJ Open. 2017;7(9):e015471.

68. Bates I, John C, Bruno A, Fu P, Aliabadi S. An analysis of the global pharmacy workforce capacity. Hum Resour Health. 2016;14(1):1-7. doi:10.1186/s12960-016-0158-z

69. Fathelrahman A, Ibrahim MI, Wertheimer A. Pharmacy Practice in Developing Countries : Achievements and Challenges. Academic Press; 2016.

70. Gagliardi AR, Alhabib S. Trends in guideline implementation: a scoping systematic review. Implement Sci. 2015;10:54. doi:10.1186/s13012-015-0247-8

71. MacKeigan LD, Ijaz N, Bojarski EA, Dolovich L. Implementation of a reimbursed medication review program: Corporate and pharmacy level strategies. Res Soc Adm Pharm. 2017;13(5):947958. doi:10.1016/j.sapharm.2017.03.057

72. Squires JE, Sullivan K, Eccles MP, Worswick J, Grimshaw JM. Are multifacted interventions more effective than single-component interventions in changing health-care professionals' behaviours? An overview of systematic reviews. Implement Sci. 2014;9(152):1-22. 
73. Shoemaker SJ, Curran GM, Swan H, Teeter BS, Thomas J. Application of the Consolidated Framework for Implementation Research to community pharmacy: A framework for implementation research on pharmacy services. Res Social Adm Pharm. 2017;13(5):905-913.

74. Moullin JC, Sabater-Hernández D, Benrimoj SI. Model for the evaluation of implementation programs and professional pharmacy services. Res Soc Adm Pharm. 2016;12(3):515-522. doi:10.1016/j.sapharm.2015.08.003

75. Lynch EA, Mudge A, Knowles S, Kitson AL, Hunter SC, Harvey G. “ There is nothing so practical as a good theory" : a pragmatic guide for selecting theoretical approaches for implementation projects. BMC Health Serv Res. 2018; 18(857): 1-11.

76. Birken SA, Powell BJ, Shea CM, et al. Criteria for selecting implementation science theories and frameworks : results from an international survey. Implement Sci. 2017; 12(124): 1-9. doi:10.1186/s13012-017-0656-y

77. Watson MC, Bond CM, Grimshaw JM, Mollison J, Ludbrook A, Walker AE. Educational strategies to promote evidence-based community pharmacy practice: a cluster randomized controlled trial (RCT). Fam Pract. 2002;19(5):529-536.

78. Franklin BD, Reynolds M, Sadler S, et al. The effect of the electronic transmission of prescriptions on dispensing errors and prescription enhancements made in English community pharmacies: A naturalistic stepped wedge study. BMJ Qual Saf. 2014;23(8):629-638. doi:10.1136/bmjqs-2013002776

79. May CR, Johnson M, Finch T. Implementation, context and complexity. Implement Sci. 2016;11(1):1-12. doi:10.1186/s13012-016-0506-3

80. Moore GF, Audrey S, Barker M, et al. Process evaluation of complex interventions: Medical Research Council guidance. BMJ. 2015;350:1-7. doi:10.1136/bmj.h1258

81. Australian Government Department of Health and Ageing. Program Specific Guidelines: Home Medication Review. 5th Community Pharm Agreem. 2013:1-8. 


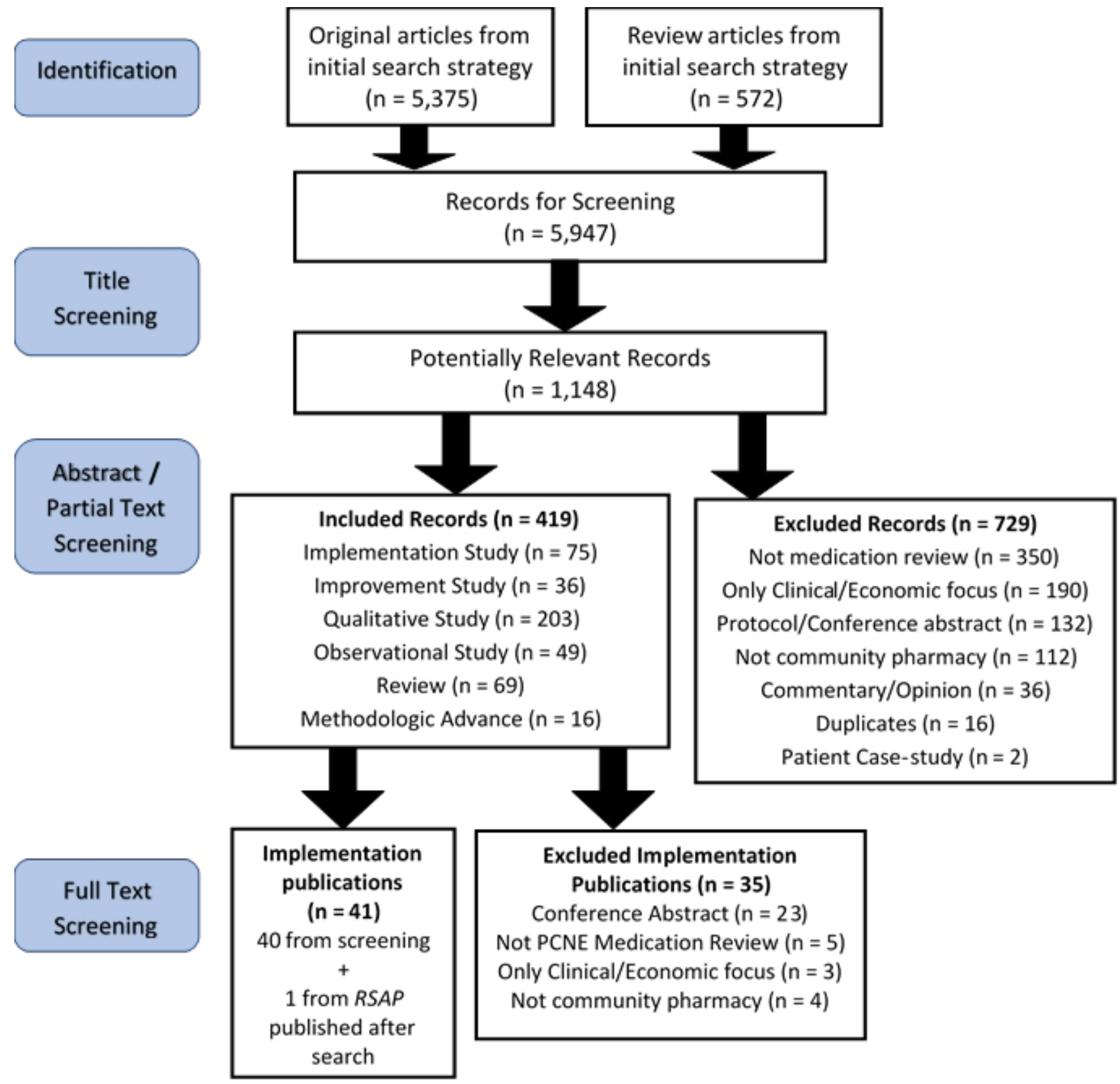


Table 1 - Categories and Relevance of Literature Comprising Scoping Review

\begin{tabular}{|c|c|c|}
\hline Article Type & \begin{tabular}{|l} 
Description \\
\end{tabular} & $\begin{array}{r}\text { Relevance } \\
\end{array}$ \\
\hline $\begin{array}{l}\text { Original Implementation } \\
\text { Intervention Study }\end{array}$ & $\begin{array}{l}\text { Studies that observe or } \\
\text { evaluate the effect of an active } \\
\text { or passive implementation } \\
\text { intervention on the delivery of } \\
\text { medication review services }\end{array}$ & $\begin{array}{l}\text { Provides evidence of what } \\
\text { strategies are effective or } \\
\text { ineffective in implementing } \\
\text { medication review services in } \\
\text { various community pharmacy } \\
\text { workplace settings }\end{array}$ \\
\hline $\begin{array}{l}\text { Original Improvement } \\
\text { Intervention Study }\end{array}$ & $\begin{array}{l}\text { Studies that observe or } \\
\text { evaluate the effect of a process } \\
\text { change to improve the } \\
\text { facilitation or quality of } \\
\text { medication reviews where the } \\
\text { service is already established }\end{array}$ & $\begin{array}{l}\text { Provides evidence of what } \\
\text { strategies are effective or } \\
\text { ineffective in improving } \\
\text { efficiency, quality or outcomes of } \\
\text { already established medication } \\
\text { review services in various } \\
\text { community pharmacy workplace } \\
\text { settings }\end{array}$ \\
\hline $\begin{array}{l}\text { Original Qualitative } \\
\text { Study }\end{array}$ & $\begin{array}{l}\text { Studies that utilize qualitative } \\
\text { methodology such as } \\
\text { interviews, focus-groups or } \\
\text { surveys that ask for } \\
\text { perspective or opinion on } \\
\text { medication reviews (or aspects } \\
\text { of medication reviews) }\end{array}$ & $\begin{array}{l}\text { Provides evidence on barriers } \\
\text { and/or facilitators to medication } \\
\text { review service implementation. } \\
\text { Also offers information on unique } \\
\text { workplace contexts not } \\
\text { measurable through traditional } \\
\text { quantitative methods (i.e } \\
\text { relationships, interprofessional } \\
\text { politics etc.) }\end{array}$ \\
\hline $\begin{array}{l}\text { Original Observational } \\
\text { Study }\end{array}$ & $\begin{array}{l}\text { Studies that evaluate trends } \\
\text { relating to medication review } \\
\text { delivery without a passive } \\
\text { intervention (i.e policy } \\
\text { change) having occurred. May } \\
\text { rely on administrative, survey } \\
\text { or other quantitative data. }\end{array}$ & $\begin{array}{l}\text { Measurement of real-world } \\
\text { patterns of medication review } \\
\text { service delivery and potentially } \\
\text { associated variables such as } \\
\text { geography, pharmacy type, pre- } \\
\text { existing arrangements and other } \\
\text { factors that may inform context } \\
\text { specific implementation strategies }\end{array}$ \\
\hline Review & $\begin{array}{l}\text { Knowledge synthesis } \\
\text { publications (i.e systematic, } \\
\text { scoping, narrative, realist, } \\
\text { umbrella and meta-synthesis } \\
\text { reviews) that answer specific } \\
\text { question(s) related to } \\
\text { medication review services. }\end{array}$ & $\begin{array}{l}\text { Provides analysis of compiled } \\
\text { evidence related to some topic of } \\
\text { importance regarding } \\
\text { implementation of medication } \\
\text { reviews in community pharmacy }\end{array}$ \\
\hline $\begin{array}{c}\text { Methodologic } \\
\text { advancement or clinical } \\
\text { practice tool }\end{array}$ & $\begin{array}{l}\text { Studies that develop, validate } \\
\text { or otherwise improve methods } \\
\text { underlying implementation or } \\
\text { improvement of medication } \\
\text { review services }\end{array}$ & $\begin{array}{l}\text { Strengthens the rigour or quality } \\
\text { of future research or service } \\
\text { delivery }\end{array}$ \\
\hline
\end{tabular}


Table 2 - Characteristics of Implementation Studies on Medication Review Services in Community Pharmacy

\begin{tabular}{|c|c|c|c|c|c|c|c|c|}
\hline $\begin{array}{l}\text { Author } \\
\text { (Year) }\end{array}$ & Nation(s) & $\begin{array}{l}\text { Medication } \\
\text { Review Sub- } \\
\text { type (PCNE) }\end{array}$ & $\begin{array}{l}\text { Active or } \\
\text { Passive } \\
\text { Intervention }\end{array}$ & $\begin{array}{l}\text { Number and Type } \\
\text { Implementation } \\
\text { Interventions } \\
\text { (EPOC) }\end{array}$ & $\begin{array}{l}\text { Level of } \\
\text { Intervention }\end{array}$ & $\begin{array}{l}\text { Use of DII } \\
\text { tools, } \\
\text { theories, } \\
\text { frameworks } \\
\text { (Stifler et al.) }\end{array}$ & $\begin{array}{l}\text { Sample Size } \\
\text { and Locations } \\
\text { of } \\
\text { Implementation }\end{array}$ & $\begin{array}{l}\text { DII Outcomes } \\
\text { (Proctor et al.) }\end{array}$ \\
\hline $\begin{array}{l}\text { Lelubre et } \\
\text { al. }{ }^{19}(2019)\end{array}$ & Belgium & $\begin{array}{l}\text { Intermediate } \\
(2 \mathrm{a})\end{array}$ & Active & $\begin{array}{l}\text { 1) Education; } \\
\text { meetings } \\
\text { 2) Communities of } \\
\text { practice } \\
\text { 3) Clinical prompts; } \\
\text { reminders, audit and } \\
\text { feedback } \\
\text { 4) Financial } \\
\text { incentive }\end{array}$ & $\begin{array}{l}\text { Pharmacy } \\
\text { level }\end{array}$ & $\begin{array}{l}\text { Yes; RE- } \\
\text { AIM and } \\
\text { FISPh }\end{array}$ & $\begin{array}{l}80 \text { pharmacies } \\
\text { ( } 25 \text { dropping out } \\
\text { before } \\
\text { completion) }\end{array}$ & $\begin{array}{l}\text { Adoption } \\
\text { Feasibility } \\
\text { Acceptability } \\
\text { Sustainability }\end{array}$ \\
\hline $\begin{array}{l}\text { Murray et } \\
\text { al. }{ }^{21}(2018)\end{array}$ & U.S & $\begin{array}{l}\text { Intermediate } \\
(2 \mathrm{a})\end{array}$ & Active & $\begin{array}{l}\text { 1) Education; } \\
\text { outreach visits, } \\
\text { materials (focused } \\
\text { on CQI) }\end{array}$ & $\begin{array}{l}\text { Pharmacy } \\
\text { level }\end{array}$ & No & $\begin{array}{l}4 \text { Grocery chain } \\
\text { pharmacies; } 11 \\
\text { staff members } \\
\text { trained }\end{array}$ & $\begin{array}{l}\text { Adoption } \\
\text { Feasibility }\end{array}$ \\
\hline $\begin{array}{l}\text { Grootendorst } \\
\text { et al. } .^{22}(2018)\end{array}$ & Canada & $\begin{array}{l}\text { Intermediate } \\
(2 \mathrm{a})\end{array}$ & $\begin{array}{l}\text { Passive; } \\
\text { observational } \\
\text { study of } \\
\text { administrative } \\
\text { data }\end{array}$ & $\begin{array}{l}\text { 1) Role expansion or } \\
\text { task-shifting; } \\
\text { Pharmacy } \\
\text { technicians }\end{array}$ & $\begin{array}{l}\text { Pharmacy } \\
\text { level }\end{array}$ & No & $\begin{array}{l}\text { All Ontario } \\
\text { pharmacies; } \\
4143 \text { registered } \\
\text { pharmacy } \\
\text { technicians }\end{array}$ & Adoption \\
\hline $\begin{array}{l}\text { Lengel et } \\
\text { al. }{ }^{23}(2018)\end{array}$ & U.S & $\begin{array}{l}\text { Intermediate } \\
(2 \mathrm{a})\end{array}$ & Active & $\begin{array}{l}\text { 1) Role expansion or } \\
\text { task-shifting; } \\
\text { pharmacy } \\
\text { technicians }\end{array}$ & $\begin{array}{l}\text { Pharmacy } \\
\text { level }\end{array}$ & No & $\begin{array}{l}32 \text { Grocery } \\
\text { chain } \\
\text { pharmacies }\end{array}$ & $\begin{array}{l}\text { Adoption } \\
\text { Acceptability }\end{array}$ \\
\hline
\end{tabular}




\begin{tabular}{|c|c|c|c|c|c|c|c|c|}
\hline $\begin{array}{l}\text { Mailleux et } \\
\text { al. }{ }^{24}(2018)\end{array}$ & U.S & Unclear & Active & $\begin{array}{l}\text { 1) Patient mediated } \\
\text { intervention; } \\
\text { educational materials }\end{array}$ & $\begin{array}{l}\text { Individual } \\
\text { level } \\
\text { (Patient) }\end{array}$ & No & 35 patients & Acceptability \\
\hline $\begin{array}{l}\text { Garcia- } \\
\text { Cardenas et } \\
\text { al. }^{25}(2017)\end{array}$ & Spain & $\begin{array}{l}\text { Intermediate } \\
(2 \mathrm{a})\end{array}$ & Active & $\begin{array}{l}\text { 1) Organizational } \\
\text { culture; work-place } \\
\text { leaders } \\
\text { 2) Health } \\
\text { information } \\
\text { technology; CQI } \\
\text { 3) Environment; re- } \\
\text { structuring lay-out } \\
\text { 4) Role expansion or } \\
\text { task-shifting; } \\
\text { pharmacy } \\
\text { technicians } \\
\text { 5) Education; } \\
\text { training }\end{array}$ & $\begin{array}{l}\text { Pharmacy } \\
\text { level }\end{array}$ & $\begin{array}{l}\text { Yes; Fixsen } \\
\text { et al. for } \\
\text { process } \\
\text { model, } \\
\text { Proctor et al. } \\
\text { for evaluation }\end{array}$ & $\begin{array}{l}\text { Single } \\
\text { pharmacy; } \\
4 \text { pharmacists } \\
2 \text { technicians }\end{array}$ & $\begin{array}{l}\text { Penetration } \\
\text { Costs } \\
\text { Feasibility } \\
\text { Fidelity } \\
\text { Acceptability } \\
\text { Appropriateness }\end{array}$ \\
\hline $\begin{array}{l}\text { Isetts }^{26} \\
(2017)\end{array}$ & U.S & Unclear & Active & $\begin{array}{l}\text { 1) Teams; referral } \\
\text { systems } \\
\text { 2) Health } \\
\text { information systems }\end{array}$ & $\begin{array}{l}\text { Health } \\
\text { system level }\end{array}$ & $\begin{array}{l}\text { Yes; } \\
\text { Continuous } \\
\text { Quality } \\
\text { Improvement } \\
\text { model } \\
\end{array}$ & 15 pharmacies & Feasibility \\
\hline $\begin{array}{l}\text { Houle et al. }{ }^{27} \\
(2017)\end{array}$ & Canada & $\begin{array}{l}\text { Intermediate } \\
(2 \mathrm{a})\end{array}$ & Active & $\begin{array}{l}\text { 1) Workplace } \\
\text { changes; } \\
\text { environment } \\
\text { 2) Continuous } \\
\text { Quality } \\
\text { Improvement }\end{array}$ & $\begin{array}{l}\text { Pharmacy } \\
\text { level }\end{array}$ & Yes; PARiHS & 10 pharmacies & Adoption \\
\hline
\end{tabular}




\begin{tabular}{|c|c|c|c|c|c|c|c|c|}
\hline $\begin{array}{l}\text { Tomaszewski et } \\
\text { al. }{ }^{28}(2017)\end{array}$ & U.S & Unclear & $\begin{array}{l}\text { Passive; } \\
\text { preference- } \\
\text { based } \\
\text { discrete } \\
\text { choice study }\end{array}$ & $\begin{array}{l}\text { 1) Financial } \\
\text { arrangement; } \\
\text { voucher schemes }\end{array}$ & $\begin{array}{l}\text { Individual } \\
\text { level } \\
\text { (Patient) }\end{array}$ & No & 72 patients & Acceptability \\
\hline $\begin{array}{l}\text { Stafford et } \\
\text { al. }{ }^{29}(2017)\end{array}$ & U.S & $\begin{array}{l}\text { Intermediate } \\
(2 \mathrm{a})\end{array}$ & Active & $\begin{array}{l}\text { 1) Financial } \\
\text { arrangement; } \\
\text { payment methods, } \\
\text { pay-for-performance } \\
\text { 2) Education; } \\
\text { meetings, outreach } \\
\text { visits, } \\
\text { 3) Clinical prompts; } \\
\text { audit and feedback, } \\
\text { reminders } \\
\text { 4) Communities of } \\
\text { practice }\end{array}$ & $\begin{array}{l}\text { Pharmacy } \\
\text { level }\end{array}$ & No & 29 pharmacies & Adoption \\
\hline $\begin{array}{l}\text { Smith et al. }{ }^{30} \\
(2017)\end{array}$ & U.S. & $\begin{array}{l}\text { Intermediate } \\
(2 \mathrm{a})\end{array}$ & Active & $\begin{array}{l}\text { 1) Health } \\
\text { information systems, } \\
\text { communication } \\
\text { technology } \\
\text { Tailored multi- } \\
\text { faceted interventions }\end{array}$ & $\begin{array}{l}\text { Health } \\
\text { system / } \\
\text { Pharmacy } \\
\text { level }\end{array}$ & No & 123 pharmacies & $\begin{array}{l}\text { Adoption } \\
\text { Feasibility } \\
\text { Acceptability }\end{array}$ \\
\hline $\begin{array}{l}\text { Vande } \\
\text { Griend }^{31} \\
(2017)\end{array}$ & U.S. & $\begin{array}{l}\text { Intermediate } \\
(2 \mathrm{a})\end{array}$ & Active & $\begin{array}{l}\text { 1) Education; } \\
\text { student involvement }\end{array}$ & $\begin{array}{l}\text { Pharmacy } \\
\text { level }\end{array}$ & No & $\begin{array}{l}35 \text { students over } \\
7 \text { months at } \\
\text { unknown } \\
\text { number of } \\
\text { pharmacy } \\
\text { locations }\end{array}$ & $\begin{array}{l}\text { Adoption } \\
\text { Cost }\end{array}$ \\
\hline
\end{tabular}




\begin{tabular}{|c|c|c|c|c|c|c|c|c|}
\hline $\begin{array}{l}\text { Pagano et } \\
\text { al. }{ }^{32}(2016)\end{array}$ & U.S & $\begin{array}{l}\text { Intermediate } \\
(2 \mathrm{a})\end{array}$ & Active & $\begin{array}{l}\text { 1) Education; } \\
\text { workshop and/or } \\
\text { video } \\
\text { 2) Continuous } \\
\text { Quality } \\
\text { Improvement }\end{array}$ & $\begin{array}{l}\text { Pharmacy } \\
\text { level }\end{array}$ & No & $\begin{array}{l}119 \text { grocery } \\
\text { store pharmacies }\end{array}$ & Adoption \\
\hline $\begin{array}{l}\text { Schoenrock } \\
\text { et al. }{ }^{33}(2016)\end{array}$ & U.S & $\begin{array}{l}\text { Intermediate } \\
(2 \mathrm{a})\end{array}$ & Active & $\begin{array}{l}\text { 1) Teams; referral } \\
\text { systems } \\
\text { 2) Workplace } \\
\text { changes; staffing } \\
\text { 3) Education; } \\
\text { workshop, } \\
\text { continuous quality } \\
\text { improvement }\end{array}$ & $\begin{array}{l}\text { Pharmacy } \\
\text { level / } \\
\text { Individual } \\
\text { level }\end{array}$ & No & $\begin{array}{l}3 \text { pharmacies; } 7 \\
\text { pharmacists }\end{array}$ & $\begin{array}{l}\text { Adoption } \\
\text { Feasibility } \\
\text { Fidelity }\end{array}$ \\
\hline $\begin{array}{l}\text { Bradley et } \\
\text { al. }^{34}(2016)\end{array}$ & U.S & $\begin{array}{l}\text { Intermediate } \\
(2 \mathrm{a})\end{array}$ & Active & $\begin{array}{l}\text { 1) Education; } \\
\text { workshop } \\
\text { 2) Teams; referral } \\
\text { systems, } \\
\text { coordination of care } \\
\text { (with physicians) }\end{array}$ & $\begin{array}{l}\text { Pharmacy / } \\
\text { Individual } \\
\text { level }\end{array}$ & No & $\begin{array}{l}2 \text { pharmacies; } 8 \\
\text { pharmacists }\end{array}$ & $\begin{array}{l}\text { Adoption } \\
\text { Acceptability }\end{array}$ \\
\hline $\begin{array}{l}\text { Huet et al. }{ }^{35} \\
(2015)\end{array}$ & U.S & Unclear & Active & $\begin{array}{l}\text { 1) Patient mediated } \\
\text { interventions; } \\
\text { promotional } \\
\text { strategies }\end{array}$ & $\begin{array}{l}\text { Individual } \\
\text { level } \\
\text { (Patient) }\end{array}$ & No & $\begin{array}{l}30 \text { grocery store } \\
\text { pharmacies }\end{array}$ & Acceptability \\
\hline $\begin{array}{l}\text { Lasota et al. }{ }^{36} \\
(2015)\end{array}$ & U.S & $\begin{array}{l}\text { Intermediate } \\
(2 \mathrm{a})\end{array}$ & Active & $\begin{array}{l}\text { 1) Continuous } \\
\text { quality improvement }\end{array}$ & $\begin{array}{l}\text { Pharmacy } \\
\text { level }\end{array}$ & No & $\begin{array}{l}3 \text { grocery store } \\
\text { pharmacies }\end{array}$ & Adoption \\
\hline
\end{tabular}




\begin{tabular}{|c|c|c|c|c|c|c|c|c|}
\hline $\begin{array}{l}\text { Lester et al. }^{37} \\
(2014)\end{array}$ & U.S & $\begin{array}{l}\text { Intermediate } \\
(2 \mathrm{a})\end{array}$ & Active & $\begin{array}{l}\text { 1) Role-expansion or } \\
\text { task-shifting; } \\
\text { pharmacists and } \\
\text { technicians }\end{array}$ & $\begin{array}{l}\text { Pharmacy } \\
\text { level }\end{array}$ & No & 1 pharmacy & $\begin{array}{l}\text { Feasibility } \\
\text { Acceptability }\end{array}$ \\
\hline $\begin{array}{l}\text { Grindrod et } \\
\text { al. }{ }^{38}(2013)\end{array}$ & Canada & $\begin{array}{l}\text { Intermediate } \\
(2 \mathrm{a})\end{array}$ & Active & $\begin{array}{l}\text { 1) Communities of } \\
\text { practice } \\
\text { 2) Education; } \\
\text { outreach visits }\end{array}$ & $\begin{array}{l}\text { Individual } \\
\text { level }\end{array}$ & No & $\begin{array}{l}4 \text { new-graduate } \\
\text { pharmacists at } 4 \\
\text { locations }\end{array}$ & $\begin{array}{l}\text { Adoption } \\
\text { Acceptability } \\
\text { Fidelity } \\
\text { Feasibility }\end{array}$ \\
\hline $\begin{array}{l}\text { Teichert et } \\
\text { al. }{ }^{39}(2013)\end{array}$ & Netherlands & $\begin{array}{l}\text { Intermediate } \\
(2 \mathrm{a})\end{array}$ & Active & $\begin{array}{l}\text { 1) Education; } \\
\text { workshops } \\
\text { 2) Practice tool } \\
\text { 3) Financial } \\
\text { arrangement; } \\
\text { payment methods, } \\
\text { pay-for-performance }\end{array}$ & $\begin{array}{l}\text { Individual } \\
\text { level }\end{array}$ & No & 56 pharmacies & $\begin{array}{l}\text { Adoption } \\
\text { Fidelity }\end{array}$ \\
\hline $\begin{array}{l}\text { Riley }^{40} \\
(2013)\end{array}$ & Canada & $\begin{array}{l}\text { Intermediate } \\
(2 \mathrm{a})\end{array}$ & Active & $\begin{array}{l}\text { 1) Role expansion } \\
\text { and task-shifting; } \\
\text { pharmacist }\end{array}$ & $\begin{array}{l}\text { Pharmacy } \\
\text { level }\end{array}$ & Yes; SWOT & $\begin{array}{l}1 \text { pharmacist at } \\
1 \text { pharmacy }\end{array}$ & $\begin{array}{l}\text { Adoption } \\
\text { Acceptability } \\
\text { Cost }\end{array}$ \\
\hline $\begin{array}{l}\text { Pattin \& } \\
\text { Szyskowski }^{41} \\
(2012)\end{array}$ & U.S & $\begin{array}{l}\text { Intermediate } \\
(2 \mathrm{a})\end{array}$ & Active & $\begin{array}{l}\text { 1) Environment } \\
\text { 2) Educational; } \\
\text { student involvement }\end{array}$ & $\begin{array}{l}\text { Pharmacy } \\
\text { level }\end{array}$ & No & $\begin{array}{l}1 \text { grocery store } \\
\text { pharmacy }\end{array}$ & Adoption \\
\hline $\begin{array}{l}\text { Houle et al. }{ }^{42} \\
(2012)\end{array}$ & Canada & $\begin{array}{l}\text { Intermediate } \\
(2 \mathrm{a})\end{array}$ & $\begin{array}{l}\text { Passive; } \\
\text { projection of } \\
\text { exploratory } \\
\text { intervention } \\
\text { using } \\
\text { observation } \\
\text { data }\end{array}$ & $\begin{array}{l}\text { 1) Environment; } \\
\text { improving triage }\end{array}$ & $\begin{array}{l}\text { Pharmacy } \\
\text { level }\end{array}$ & No & $\begin{array}{l}\text { Data from } 341 \\
\text { pharmacies }\end{array}$ & $\begin{array}{l}\text { Adoption } \\
\text { Cost }\end{array}$ \\
\hline
\end{tabular}




\begin{tabular}{|c|c|c|c|c|c|c|c|c|}
\hline $\begin{array}{l}\text { Lam et al. }{ }^{43} \\
\text { (2012) }\end{array}$ & U.S & $\begin{array}{l}\text { Intermediate } \\
(2 a)\end{array}$ & Active & $\begin{array}{l}\text { 1) Communities of } \\
\text { practice; academia } \\
\text { and community } \\
\text { pharmacy } \\
\text { partnership }\end{array}$ & $\begin{array}{l}\text { Health } \\
\text { system / } \\
\text { Pharmacy } \\
\text { level }\end{array}$ & No & 6 pharmacies & $\begin{array}{l}\text { Adoption } \\
\text { Acceptability } \\
\text { Cost }\end{array}$ \\
\hline $\begin{array}{l}\text { Ross \& } \\
\text { Bloodworth } \\
(2012)\end{array}$ & U.S & $\begin{array}{l}\text { Intermediate } \\
(2 \mathrm{a})\end{array}$ & Active & $\begin{array}{l}\text { 1) Education; } \\
\text { workshops } \\
\text { 2) Practice tools } \\
\text { 3) Outreach visits } \\
\text { 4) Health } \\
\text { information systems }\end{array}$ & $\begin{array}{l}\text { Health } \\
\text { system / } \\
\text { Pharmacy } \\
\text { level }\end{array}$ & No & $\begin{array}{l}13 \text { pharmacies; } \\
78 \text { pharmacists } \\
\text { and } 177 \text { student } \\
\text { pharmacists }\end{array}$ & Adoption \\
\hline $\begin{array}{l}\text { Schuh et al. }{ }^{45} \\
(2011)\end{array}$ & U.S & $\begin{array}{l}\text { Intermediate } \\
\text { (2a) }\end{array}$ & Active & $\begin{array}{l}\text { 1) Education; } \\
\text { training } \\
\text { 2) Teams; referral } \\
\text { systems, } \\
\text { coordination of care } \\
\text { 3) Communities of } \\
\text { practice; local } \\
\text { opinion leaders, } \\
\text { academic partnership }\end{array}$ & $\begin{array}{l}\text { Pharmacy } \\
\text { level }\end{array}$ & No & $\begin{array}{l}1 \text { pharmacy } \\
\text { (next to a multi- } \\
\text { specialty clinic) }\end{array}$ & Sustainability \\
\hline $\begin{array}{l}\text { Dualde et } \\
\text { al. }{ }^{46}(2009)\end{array}$ & Spain & Unclear & Active & $\begin{array}{l}\text { 1) Education; } \\
\text { meetings, video- } \\
\text { conferencing courses }\end{array}$ & $\begin{array}{l}\text { Individual } \\
\text { level }\end{array}$ & $\begin{array}{l}\text { Yes; Roger's } \\
\text { diffusion of } \\
\text { innovation }\end{array}$ & 225 pharmacists & $\begin{array}{l}\text { Adoption } \\
\text { Acceptability }\end{array}$ \\
\hline $\begin{array}{l}\text { Trapskin et } \\
\text { al. }{ }^{47}(2009)\end{array}$ & U.S & $\begin{array}{l}\text { Intermediate } \\
(2 \mathrm{a})\end{array}$ & Passive & $\begin{array}{l}\text { 1) Financial } \\
\text { arrangement; fee- } \\
\text { for-service, pay-for- } \\
\text { performance }\end{array}$ & $\begin{array}{l}\text { Health } \\
\text { system level }\end{array}$ & No & $\begin{array}{l}53 \text { pharmacies; } \\
106 \text { pharmacists, } \\
45 \text { pharmacy } \\
\text { students, } 6 \\
\text { technicians }\end{array}$ & $\begin{array}{l}\text { Adoption } \\
\text { Cost }\end{array}$ \\
\hline
\end{tabular}




\begin{tabular}{|c|c|c|c|c|c|c|c|c|}
\hline $\begin{array}{l}\text { Lewis et al. }{ }^{48} \\
(2008)\end{array}$ & U.S & $\begin{array}{l}\text { Intermediate } \\
(2 \mathrm{a})\end{array}$ & Active & $\begin{array}{l}\text { 1) Education; } \\
\text { meetings } \\
\text { 2) Marketing; } \\
\text { outreach visits, } \\
\text { materials } \\
\text { 3) Communities of } \\
\text { practice; academia } \\
\text { and community } \\
\text { pharmacies }\end{array}$ & $\begin{array}{l}\text { Health } \\
\text { system / } \\
\text { pharmacy } \\
\text { level }\end{array}$ & No & $\begin{array}{l}30 \text { pharmacists } \\
\text { ( } 70 \% \text { in } \\
\text { community } \\
\text { pharmacies) }\end{array}$ & $\begin{array}{l}\text { Adoption } \\
\text { Fidelity }\end{array}$ \\
\hline $\begin{array}{l}\text { Blenkinsopp } \\
\text { et al. } 49,50 \\
(2007 \& \\
2010)\end{array}$ & U.K & $\begin{array}{l}\text { Intermediate } \\
(2 \mathrm{a}) \\
\text { Advanced }\end{array}$ & Passive & $\begin{array}{l}\text { 1) Financial } \\
\text { arrangement; fee- } \\
\text { for-service (national } \\
\text { policy) }\end{array}$ & $\begin{array}{l}\text { Health } \\
\text { system level }\end{array}$ & $\begin{array}{l}\text { Yes; Roger's } \\
\text { diffusion of } \\
\text { innovation }\end{array}$ & $\begin{array}{l}10 \% \text { of all } \\
\text { community } \\
\text { pharmacies }\end{array}$ & Adoption \\
\hline $\begin{array}{l}\text { Rouleau et } \\
\text { al. }{ }^{51}(2007)\end{array}$ & Canada & Simple (1) & Active & $\begin{array}{l}\text { 1) Education; } \\
\text { meetings, materials }\end{array}$ & $\begin{array}{l}\text { Individual } \\
\text { level }\end{array}$ & No & $\begin{array}{l}27 \text { pharmacies; } \\
48 \text { pharmacists }\end{array}$ & Adoption \\
\hline $\begin{array}{l}\text { Jaffray et } \\
\text { al. } .^{52}(2007)\end{array}$ & England & Unclear & Active & $\begin{array}{l}\text { 1) Education; } \\
\text { meetings }\end{array}$ & $\begin{array}{l}\text { Individual } \\
\text { level }\end{array}$ & No & 102 pharmacists & $\begin{array}{l}\text { Acceptability } \\
\text { Feasibility }\end{array}$ \\
\hline $\mathrm{Pai}^{53}(2005)$ & U.S. & $\begin{array}{l}\text { Intermediate } \\
(2 \mathrm{a})\end{array}$ & Active & $\begin{array}{l}\text { 1) Role expansion or } \\
\text { task-shifting; } \\
\text { workflow re-design }\end{array}$ & $\begin{array}{l}\text { Pharmacy } \\
\text { level }\end{array}$ & No & $\begin{array}{l}1 \text { pharmacy; } \\
\text { clinical } \\
\text { community } \\
\text { pharmacist }\end{array}$ & Adoption \\
\hline $\begin{array}{l}\text { Stuurman- } \\
\text { Bieze }^{54} \\
(2005)\end{array}$ & Netherlands & $\begin{array}{l}\text { Intermediate } \\
(2 \mathrm{a})\end{array}$ & Active & $\begin{array}{l}\text { 1) Education; } \\
\text { meetings, materials, } \\
\text { 2) Health } \\
\text { information } \\
\text { technology }\end{array}$ & $\begin{array}{l}\text { Individual } \\
\text { level }\end{array}$ & No & $\begin{array}{l}24 \text { pharmacies; } \\
27 \text { pharmacists }\end{array}$ & $\begin{array}{l}\text { Adoption } \\
\text { Acceptability } \\
\text { Feasibility }\end{array}$ \\
\hline
\end{tabular}




\begin{tabular}{|c|c|c|c|c|c|c|c|c|}
\hline $\begin{array}{l}\text { Aston \& } \\
\text { Black }^{55} \\
(2005)\end{array}$ & U.K & $\begin{array}{l}\text { Intermediate } \\
(2 \mathrm{a}) \\
\text { Advanced }\end{array}$ & Active & $\begin{array}{l}\text { 1) Education; } \\
\text { advanced } \\
\text { qualification or } \\
\text { certification }\end{array}$ & $\begin{array}{l}\text { Individual } \\
\text { level }\end{array}$ & No & 227 pharmacists & $\begin{array}{l}\text { Adoption } \\
\text { Acceptability }\end{array}$ \\
\hline $\begin{array}{l}\text { Lopatka \& } \\
\text { Bachynsky } 56 \\
(2004)\end{array}$ & Canada & Unclear & Active & $\begin{array}{l}\text { 1) Financial } \\
\text { arrangement; } \\
\text { alternative } \\
\text { reimbursement } \\
\text { structure } \\
\text { 2) Educational; } \\
\text { meetings }\end{array}$ & $\begin{array}{l}\text { Health- } \\
\text { system level }\end{array}$ & $\begin{array}{l}\text { Yes; Raisch } \\
\text { model on } \\
\text { barriers to } \\
\text { providing } \\
\text { cognitive } \\
\text { services* }\end{array}$ & 112 pharmacists & $\begin{array}{l}\text { Adoption } \\
\text { Acceptability }\end{array}$ \\
\hline $\begin{array}{l}\text { Carter et al. }{ }^{57} \\
(2003)\end{array}$ & U.S & $\begin{array}{l}\text { Intermediate } \\
(2 \mathrm{a})\end{array}$ & Active & $\begin{array}{l}\text { 1) Financial } \\
\text { arrangement; fee- } \\
\text { for-service } \\
\text { 2) Education; } \\
\text { meetings }\end{array}$ & $\begin{array}{l}\text { Health- } \\
\text { system / } \\
\text { Pharmacy } \\
\text { level }\end{array}$ & No & $\begin{array}{l}117 \text { community } \\
\text { pharmacies }\end{array}$ & $\begin{array}{l}\text { Adoption } \\
\text { Feasibility } \\
\text { Fidelity }\end{array}$ \\
\hline $\begin{array}{l}\text { March et al. }{ }^{58} \\
\text { (1999) }\end{array}$ & Australia & $\begin{array}{l}\text { Intermediate } \\
\text { (2a) } \\
\text { Advanced }\end{array}$ & Active & $\begin{array}{l}\text { 1) Education; } \\
\text { workshops } \\
\text { 2) Communities of } \\
\text { practice; local } \\
\text { opinion leaders, } \\
\text { stakeholder } \\
\text { involvement } \\
\text { (physicians, patients) }\end{array}$ & $\begin{array}{l}\text { Pharmacy } \\
\text { level }\end{array}$ & $\begin{array}{l}\text { Yes; } \\
\text { Participatory } \\
\text { Action } \\
\text { Research } \\
\text { model }\end{array}$ & $\begin{array}{l}5 \text { pharmacies; } \\
\text { unclear number } \\
\text { of pharmacists }\end{array}$ & $\begin{array}{l}\text { Adoption } \\
\text { Acceptability }\end{array}$ \\
\hline $\begin{array}{l}\text { Barner et al. }{ }^{59} \\
\text { (1999) }\end{array}$ & U.S & Unclear & Active & $\begin{array}{l}\text { 1) Education; } \\
\text { advanced } \\
\text { qualification or } \\
\text { certification }\end{array}$ & $\begin{array}{l}\text { Individual } \\
\text { level }\end{array}$ & No & 36 pharmacists & Acceptability \\
\hline
\end{tabular}




\begin{tabular}{|l|l|l|l|l|l|l|l|}
\hline $\begin{array}{l}\text { Chen et al. }{ }^{(1999)} \\
(1999)\end{array}$ & Australia & $\begin{array}{l}\text { Intermediate } \\
(2 \mathrm{~b}) ; \\
\text { medication } \\
\text { review with } \\
\text { physicians } \\
\text { (no patient } \\
\text { involvement) }\end{array}$ & Active & $\begin{array}{l}\text { 1) Education; } \\
\text { workshops }\end{array}$ & $\begin{array}{l}\text { Individual } \\
\text { level }\end{array}$ & No & 2) Teams; physician \\
collaboration & Appropriateness \\
Anacists & & & \\
\hline
\end{tabular}

CQI; Continuous Quality Improvement, DII; Dissemination, Implementation, Improvement FISPh; Framework for Implementation Strategies in Pharmacy, PARiHS; Promoting Action on Research Implementation in Health Services, RE-AIM; Reach, Effectiveness, Adoption, Implementation, Maintenance, SWOT; Strengths, Weaknesses, Opportunities and Threats 
PRESS Guideline - Search Submission \& Peer Review Assessment

SEARCH SUBMISSION: THIS SECTION TO BE FILLED IN BY THE SEARCHER

Systematic Review Title:

Implementation of Medication Review Services in Community Pharmacies: a Scoping Review

This search strategy is ...

\begin{tabular}{|l|l|}
\hline$\sqrt{ }$ & My PRIMARY (core) database strategy - First time submitting a strategy for search question and database \\
\hline $\begin{array}{l}\text { My PRIMARY (core) strategy - Follow-up review NOT the first time submitting a strategy for search } \\
\text { question and database. If this is a response to peer review, itemize the changes made to the review } \\
\text { suggestions }\end{array}$ & SECONDARY search strategy - First time submitting a strategy for search question and database \\
& $\begin{array}{l}\text { SECONDARY search strategy - NOT the first time submitting a strategy for search question and database. If } \\
\text { this is a response to peer review, itemize the changes made to the review suggestions }\end{array}$ \\
\hline
\end{tabular}

Database

(i.e., MEDLINE,CINAHL...):

[mandatory]

MEDLINE

\section{Interface}

(i.e., Ovid, EBSCO...):

[mandatory]

Ovid 


\section{Research Question}

(Describe the purpose of the search)

[mandatory]

a) What types of KT/Implementation interventions have been undertaken (and where?) to improve the implementation and sustainability of medication review services from community pharmacists?

b) What has been learned in regards to barriers and facilitators to implementation of medication review services by community pharmacists in their practice settings?

\section{PICO Format}

(Outline the PICOs for your question - i.e., Patient, Intervention, Comparison, Outcome, and Study Design - as applicable)

PICOS Format thought to be of less relevance to us because project is scoping review?

\begin{tabular}{|c|l|}
\hline P & Community Pharmacists / Patients \\
\hline I & Diverse KT Interventions \\
\hline C & Any \\
\hline O & $\begin{array}{l}\text { Many - improvement of pharmacist, patient and health system indicators related to MTM } \\
\text { implementation }\end{array}$ \\
\hline S & Any \\
\hline
\end{tabular}

\section{Inclusion Criteria}

(List criteria such as age groups, study designs, etc., to be included) [optional]

Community pharmacy settings and medication therapy management/review services 


\section{Exclusion Criteria}

(List criteria such as study designs, date limits, etc., to be excluded) [optional]

\section{Plan is to apply this exclusion criteria during title and abstract screening}

No study design exclusions

Articles focusing solely on patient health outcomes of MTM or Medication Review services and NOT on implementation.

Articles involving community pharmacist MTM or medication review OUTSIDE of community pharmacy settings (i.e home visits or LTC facilities)

Articles focusing on MTM/Medication Review in any way that is of limited or no relevance to implementation, barriers or facilitators to implementation

Articles focusing on implementation of other clinical pharmacy services UNLESS article is a knowledge synthesis deemed of relevance to inform the scoping review

Was a search filter applied?

No

Other notes or comments you feel would be useful for the peer reviewer? [optional]

1. It was decided to leave the KT aspect of the search to the screening process. KT is such an amorphous topic that I was concerned adding in the KT aspect would lead to many articles being left out. The HIRU filter for KT always seems to vague to truly capture all the potential KT interventions out there. It was thought that just focusing on the community pharmacy and medication review service concepts would be limiting enough.

2. Core elements of MTM pulled from https://www.pharmacist.com/sites/default/files/files/core_elements_of_an_mtm_pract ice.pdf

3. Earlier versions of the searches played around with MeSH and keywords related to general practice and primary care in relation to pharmacist, as many of these interactions would take place in a community pharmacy setting. However, result sets went up significantly when these were added in.

4. Current search focused on either community pharmacy OR changing roles of pharmacy staff AND MTM

5. Current search locates 15 of 20 pre-identified studies.

6. Keyword search of just community/ies and various of pharmacy/ies found to be too broad. Need to use adj to make them relevant.

7. Potential plan to conduct secondary search strategy, relaxing the search strategy while simultaneously limiting to review articles to ensure capture of all relevant knowledge syntheses. This would conceivably increase our pre-identified study capture rate to $18 / 20$. 
Please copy and paste your search strategy here, exactly as run, including the number of hits per line. [mandatory]

Search Strategy:

Database: Ovid MEDLINE(R) Epub Ahead of Print, In-Process \& Other Non-Indexed Citations, Ovid MEDLINE(R) Daily and Ovid MEDLINE(R) $<1946$ to Present $>$

Pre-Identified Articles:

("17452664" or "18595820" or "24071523" or "27459951" or "23322285" or "28877940" or "29221929" or "28595895" or "28666816" or "27053276" or "26658232" or "27423785" or "28214149" or "29039899" or "28462792" or "29033347" or "28420595" or "28733156" or "27061142" or "27498311").ui.

1 Community Pharmacy Services/ (3894)

2 (communit* adj5 (pharmacy or pharmacies or pharmacist*)).tw,kf. (6067)

3 (pharmacist* adj5 (front line or "one to one" or face to face or upstream)).tw,kf. (119)

4 or/1-3 (7663) [Annotation: Community Pharmacy]

5 Pharmacy/ or Pharmacy Technicians/ or Pharmacists/ (25457)

6 pharmacist*.tw,kf. (27542)

7 ((pharmacist* or pharmacy) adj3 (aide or aides or assistant* or staff or technician*)).tw,kf. (2293)

8 or/5-7 (42407) [Annotation: Pharmacy Staff]

9 community health services/ or community mental health services/ or community networks/ (53767)

108 and 9 (221) [Annotation: Community Pharmacies and staff (MeSH mostly)

11 Professional Role/ (11573)

12 ((role* or scope*) adj2 (expand* or expansion* or extend* or extension* or chang*)).tw,kf. (14659)

1311 or 12 (25888) [Annotation: Role Expansion] 
148 and 13 (3386) [Annotation: Pharmacy Staff and Role Expansion]

15 Medication Therapy Management/ (1577)

16 exp Drug Prescriptions/ (30186)

17 "Drug Utilization Review"/ (3529)

18 Medication Adherence/ (14997)

19 Medication Reconciliation/ (814)

20 ((medication* or medicine* or drug*) adj3 (manag* or review* or reconcil* or plan* or record* or adher* or concord* or assess*)).tw,kf. (69037)

21 (cognitive adj3 service*).tw,kf. (355)

22 care plan*.tw,kf. (12677)

23 or/15-22 (121769) [Annotation: MTM]

244 or 10 or 14 (10075) [Annotation: Community Pharmacies AND Community Pharmacies [MeSH] AND Changing Roles]

2523 and 24 (2905) [Annotation: Community Pharm or Roles AND MTM]

26 remove duplicates from 25 (2880)

27 limit 26 to english language (2745) 


\section{PEER REVIEW ASSESSMENT: THIS SECTION TO BE FILLED IN BY THE REVIEWER}

1. TRANSLATION

\begin{tabular}{|l|l|}
\hline A ---No revisions & $X$ \\
\hline B --- Revision(s) suggested & $\square$ \\
\hline C --- Revision(s) required & $\square$ \\
\hline
\end{tabular}

If "B" or "C," please provide an explanation or example:

\section{BOOLEAN AND PROXIMITY OPERATORS}

\begin{tabular}{|l|l|}
\hline A ---No revisions & $X$ \\
\hline B --- Revision(s) suggested & $\square$ \\
\hline C --- Revision(s) required & $\square$ \\
\hline
\end{tabular}

If "B" or "C," please provide an explanation or example:

\section{SUBJECT HEADINGS}

\begin{tabular}{|l|l|}
\hline A ---No revisions & {[} \\
\hline B --- Revision(s) suggested & $X$ \\
\hline C --- Revision(s) required & {[} \\
\hline
\end{tabular}

If "B" or "C," please provide an explanation or example:

To Pharmacy Staff section:

- PHARMACIES/ (scope note: Facilities for the preparation and dispensing of drugs.)

-PHARMACEUTICAL SERVICES/

\section{TEXT WORD SEARCHING}

\begin{tabular}{|l|l|}
\hline A ---No revisions & {[} \\
\hline B --- Revision(s)suggested & $X$ \\
\hline C --- Revision(s) required & {[} \\
\hline
\end{tabular}

If "B" or "C," please provide an explanation or example:

- I found a few papers that included "community pharmaceutical care" - not sure if this would fit your inclusion criteria/worth exploring in a text word search?

- MTM.tw,kf. 


\section{SPELLING, SYNTAX, AND LINE NUMBERS}

\begin{tabular}{|l|l|}
\hline A ---No revisions & $X$ \\
\hline B --- Revision(s)suggested & $\square$ \\
\hline C --- Revision(s) required & $\square$ \\
\hline
\end{tabular}

If "B" or "C," please provide an explanation or example:

\section{LIMITS AND FILTERS}

\begin{tabular}{|l|l|}
\hline A ---No revisions & X \\
\hline B -- Revision(s) suggested & $\square$ \\
\hline C --- Revision(s) required & $\square$ \\
\hline
\end{tabular}

If "B" or "C," please provide an explanation or example:

OVERALL EVALUATION (Note: If one or more "revision required" is noted above, the response below must be "revisions required".)

\begin{tabular}{|l|l|}
\hline A ---No revisions & $\square$ \\
\hline B --- Revision(s) suggested & $X$ \\
\hline C --- Revision(s) required & $\square$ \\
\hline
\end{tabular}

Additional comments:

Looks good - Agree with not trying to include a KT section. Minor revisions suggested - only a few Mesh and text words that could be added, but I don't think they're going to create a drastic impact in your search results. 


\title{
Bibliographic Lists by Category: Medication Review Implementation in Community Pharmacy
}

\author{
Table of Contents \\ i) Review Articles - pg. 1-5 \\ ii) Qualitative Studies - pg. 6-21 \\ iii) Observational / Descriptive Studies - pg. 22-25 \\ iv) Improvement Studies - pg. 26-28 \\ v) Methodologic Advances / Practice Tools - pg. 29-30

\section{i) Review Articles}

1. Luetsch K. Attitudes and attributes of pharmacists in relation to practice change - A scoping review and discussion. Res Soc Adm Pharm. 2017;13(3):440-455.e11. doi:10.1016/j.sapharm.2016.06.010.

2. Butler A, Dehner M, Gates RJ, et al. Comprehensive Medication Management programs: 2015 status in Southern California. Res Social Adm Pharm. 2017;13(1):63-87.

3. Shoemaker SJ, Curran GM, Swan H, Teeter BS, Thomas J. Application of the Consolidated Framework for Implementation Research to community pharmacy: A framework for implementation research on pharmacy services. Res Soc Adm Pharm. 2017;13(5):905-913. doi:10.1016/j.sapharm.2017.06.001.

4. Mrhar A. Translation of clinical pharmacy research into practice: A Slovenian case. Acta Pharm Hung. 2017;87(3):129.

5. Aston J, Huynh C, Sinclair A, Wilson K, Terry D. Medication Review of Children on Long Term Medications: A Review of the Literature. Arch Dis Child. 2016;101(9):e2..

6. Luetsch K. Pharmacists' personality traits-barriers to practice change? Int J Pharm Pract. 2016;24:19.

7. Jokanovic N, Tan ECK, van den Bosch D, Kirkpatrick CM, Dooley MJ, Bell JS. Clinical medication review in Australia: A systematic review. Res Social Adm Pharm. 2016;12(3):384418.

8. Swieczkowski D, Poniatowski P, Merks P, Jaguszewski M. The pharmaceutical care in asthma Polish and global perspective. Pneumonol Alergol Pol. 2016;84(4):225-231.

9. Rosenthal M, Holmes E, Banahan B. 3rd. Making MTM implementable and sustainable in community pharmacy: Is it time for a different game plan? Res Social Adm Pharm. 2016;12(3):523-528. 
10. Sadek MM, Elnour AA, Al Kalbani NMS, et al. Community pharmacy and the extended community pharmacist practice roles: The UAE experiences. Saudi Pharm J SPJ Off Publ Saudi Pharm Soc. 2016;24(5):563-570.

11. Hersberger KE, Messerli M. Development of Clinical Pharmacy in Switzerland: Involvement of Community Pharmacists in Care for Older Patients. Drugs Aging. 2016;33(3):205-211.

12. Lynum KSB, Hill AM. Psychiatric services: a platform for MTM. J Pharm Pract. 2015;28(1):1320.

13. Smalls TD, Broughton AD, Hylick E V, Woodard TJ. Providing medication therapy management for smoking cessation patients. J Pharm Pract. 2015;28(1):21-25.

14. Bardet J-D, Vo T-H, Bedouch P, Allenet B. Physicians and community pharmacists collaboration in primary care: A review of specific models. Res Social Adm Pharm. 2015;11(5):602-622.

15. Glenn ZM, Mahdavian SL, Woodard TJ. Preparing to provide MTM services. J Pharm Pract. 2015;28(1):6-9.

16. Scahill S, Fowler JL, Hattingh HL, Kelly F, Wheeler AJ. Mapping the terrain: A conceptual schema for a mental health medication support service in community pharmacy. SAGE open Med. 2015;3:2050312115603002.

17. Martins SF, van Mil JWF, da Costa FA. The organizational framework of community pharmacies in Europe. Int J Clin Pharm. 2015;37(5):896-905.

18. Houle SKD, Grindrod KA, Chatterley T, Tsuyuki RT. Paying pharmacists for patient care: A systematic review of remunerated pharmacy clinical care services. Can Pharm J (Ott). 2014;147(4):209-232..

19. Pammett R, Jorgenson $D$. Review of community pharmacy based medication review services in Canada. Int J Pharm Pract. 2014;22:46-47.

20. Rubio-Valera M, Chen TF, O'Reilly CL. New roles for pharmacists in community mental health care: a narrative review. Int J Environ Res Public Health. 2014;11(10):10967-10990.

21. White CM. Pharmacists need recognition as providers to enhance patient care. Ann Pharmacother. 2014;48(2):268-273.

22. Willeboordse F, Hugtenburg JG, Schellevis FG, Elders PJM. Patient participation in medication reviews is desirable but not evidence-based: A systematic literature review. Br J Clin Pharmacol. 2014;78(6):1201-1216.

23. Fang Y, Yang S, Zhou S, Jiang M, Liu J. Community pharmacy practice in China: past, present and future. Int J Clin Pharm. 2013;35(4):520-528. 
24. Mossialos E, Naci H, Courtin E. Expanding the role of community pharmacists: policymaking in the absence of policy-relevant evidence? Health Policy. 2013;111(2):135-148.

25. Hilsenrath P, Woelfel J, Shek A, Ordanza K. Redefining the role of the pharmacist: medication therapy management. J Rural Health. 2012;28(4):425-430.

26. Gruber J. Medication therapy management: a challenge for pharmacists. Consult Pharm. 2012;27(11):782-796.

27. Isetts BJ. Pharmaceutical care, MTM, \& payment: the past, present, \& future. Ann Pharmacother. 2012;46(4):S47-56.

28. Amin M, Patwardhan P, Chewning B. Interventions to enhance community pharmacists' cognitive services: A systematic review. J Am Pharm Assoc. 2012;52(2):263-264.

29. Kauffman Y, Nair V, Herist K, Thomas V, Weidle PJ. HIV medication therapy management services in community pharmacies. J Am Pharm Assoc (2003). 2012;52(6):e287-291.

30. Hasan S, Sulieman H, Chapman CB, Stewart K, Kong DCM. Community pharmacy services in the United Arab Emirates. Int J Pharm Pract. 2012;20(4):218-225.

31. Oladapo AO, Rascati KL. Review of survey articles regarding medication therapy management (MTM) services/programs in the United States. J Pharm Pract. 2012;25(4):457470.

32. McDonough RP. Medication therapy management and collaborative practice: How to make it work. Drug Topics. 2011;155(12):52-59.

33. Clark TR, Gruber J, Martin H. Introduction to the medication regimen review--Part 1. Consult Pharm. 2010;25(11):710-720.

34. Benrimoj SI, Feletto E, Gastelurrutia MA, Martinez Martinez F, Faus MJ. A holistic and integrated approach to implementing cognitive pharmaceutical services. Ars Pharm. 2010;51(2):69-88.

35. Kaae S, Traulsen JM, Sondergaard B, Haugbolle LS. The relevance of political prestudies for implementation studies of cognitive services in community pharmacies. Res Social Adm Pharm. 2009;5(2):189-194.

36. Naik Panvelkar P, Saini B, Armour C. Measurement of patient satisfaction with community pharmacy services: a review. Pharm World Sci. 2009;31(5):525-537.

37. McKnight AG, Thomason AR. Pharmacists' advancing roles in drug and disease management: a review of states' legislation. J Am Pharm Assoc (2003). 2009;49(4):554-558.

38. Scahill S, Harrison J, Carswell P, Babar Z-U-D. Organisational culture: an important concept for pharmacy practice research. Pharm World Sci. 2009;31(5):517-521. 
39. Basak SC, Sathyanarayana D. Community pharmacy practice in India: past, present and future. South Med Rev. 2009;2(1):11-14..

40. Blenkinsopp A, Bond CM. The potential and pitfalls of medicine management: What have we learned so far? Dis Manag Heal Outcomes. 2008;16(2):79-86.

41. American Pharmacists A, of Chain Drug Stores F. Medication therapy management in pharmacy practice: core elements of an MTM service model (version 2.0). J Am Pharm Assoc (2003). 2008;48(3):341-353.

42. Alvarez-Risco A, van Mil JWF. Pharmaceutical care in community pharmacies: practice and research in Peru. Ann Pharmacother. 2007;41(12):2032-2037.

43. Buffington DE. Future of medication therapy management services in delivering patientcentered care. Am J Health Syst Pharm. 2007;64(15):S10-S23.

44. Chen TF, de Almeida Neto AC. Exploring elements of interprofessional collaboration between pharmacists and physicians in medication review. Pharm World Sci. 2007;29(6):574576.

45. Wertheimer Al, Huang WF. Opportunities for pharmacists to offer value-added services. Drug Benefit Trends. 2007;19(6):231-236.

46. Noyce PR. Providing patient care through community pharmacies in the UK: policy, practice, and research. Ann Pharmacother. 2007;41(5):861-868.

47. Herborg H, Sorensen EW, Frokjaer B. Pharmaceutical care in community pharmacies: practice and research in Denmark. Ann Pharmacother. 2007;41(4):681-689.

48. Eickhoff C, Schulz M. Pharmaceutical care in community pharmacies: practice and research in Germany. Ann Pharmacother. 2006;40(4):729-735.

49. Christensen DB, Farris KB. Pharmaceutical care in community pharmacies: practice and research in the US. Ann Pharmacother. 2006;40(7):1400-1406.

50. Costa S, Santos C, Silveira J. Community pharmacy services in Portugal. Ann Pharmacother. 2006;40(12):2228-2234.

51. Westerlund LT, Bjork HT. Pharmaceutical care in community pharmacies: practice and research in Sweden. Ann Pharmacother. 2006;40(6):1162-1169.

52. Guignard E, Bugnon O. Pharmaceutical care in community pharmacies: practice and research in Switzerland. Ann Pharmacother. 2006;40(3):512-517.

53. Roberts AS, Benrimoj SI, Chen TF, Williams KA, Aslani P. Implementing cognitive services in community pharmacy: A review of models and frameworks for change. Int J Pharm Pract. 2006;14(2):105-113. 
54. Jones EJM, Mackinnon NJ, Tsuyuki RT. Pharmaceutical care in community pharmacies: practice and research in Canada. Ann Pharmacother. 2005;39(9):1527-1533.

55. Farris KB, Fern, ez-Llimos F, Benrimoj SIC. Pharmaceutical care in community pharmacies: practice and research from around the world. Ann Pharmacother. 2005;39(9):1539-1541.

56. Burns A. Medication therapy management services: A critical review. J Am Pharm Assoc. 2005;45(5):580-587.

57. Gastelurrutia MA, Faus MJ, Fernandez-Llimos F. Providing patient care in community pharmacies in Spain. Ann Pharmacother. 2005;39(12):2105-2110.

58. Guirguis LM, Chewning BA. Role theory: literature review and implications for patientpharmacist interactions. Res Social Adm Pharm. 2005;1(4):483-507.

59. Benrimoj SI, Roberts AS. Providing patient care in community pharmacies in Australia. Ann Pharmacother. 2005;39(11):1911-1917.

60. Anderson C, Blenkinsopp A, Armstrong M. Feedback from community pharmacy users on the contribution of community pharmacy to improving the public's health: a systematic review of the peer reviewed and non-peer reviewed literature 1990-2002. Health Expect. 2004;7(3):191-202.

61. Snella KA, Trewyn RR, Hansen LB, Bradberry JC. Pharmacist compensation for cognitive services: focus on the physician office and community pharmacy. Pharmacotherapy. 2004;24(3):372-388.

62. Smith F. Community pharmacy in Ghana: enhancing the contribution to primary health care. Health Policy Plan. 2004;19(4):234-241. 9.

63. Advancing pharmacy practice through research: a 2004 perspective. J Am Pharm Assoc (2003). 2004;44(5):621-628.

64. Martin-Calero MJ, Machuca M, Murillo MD, Cansino J, Gastelurrutia MA, Faus MJ. Structural process and implementation programs of pharmaceutical care in different countries. Curr Pharm Des. 2004;10(31):3969-3985.

65. Cooksey JA, Knapp KK, Walton SM, Cultice JM. Challenges to the pharmacist profession from escalating pharmaceutical demand. Health Aff (Millwood). 2002;21(5):182-188.

66. Anderson S. The state of the world's pharmacy: a portrait of the pharmacy profession. J Interprof Care. 2002;16(4):391-404. 


\section{ii) Qualitative Studies}

1. Cardosi L, Hohmeier KC, Fisher C, Wasson M. Patient Satisfaction With a Comprehensive Medication Review Provided by a Community Pharmacist. J Pharm Technol. 2018;34(2):48-53.

2. Zhang Y, Doucette W. Understanding consumers' decision making for using a comprehensive medication review (CMR) service: A mail survey. J Am Pharm Assoc. 2018;58(3):e195.

3. Fleming M, Pourarsalan H, Chao J, Thornton J. Community chain pharmacists' barriers to providing medication therapy management and awareness of pharmacy quality alliance measures. J Am Pharm Assoc. 2018;58(3):e94-e95.

4. Cardwell K, Hughes CM, Ryan C. Community pharmacists' views of using a screening tool to structure medicines use reviews for older people: findings from qualitative interviews. Int J Clin Pharm. 2018.

5. Adeoye O, Snyder M, Lake L, Lourens S, Morris R. Association between behaviors and attitudes of pharmacy technicians and medication therapy management (MTM) completion rates. J Am Pharm Assoc. 2018;58(3):e85.

6. Patton SJ, Miller FA, Abrahamyan L, Rac VE. Expanding the clinical role of community pharmacy: A qualitative ethnographic study of medication reviews in Ontario, Canada. Health Policy. 2018;122(3):256-262.

7. Nabergoj Makovec U, Kos M, Pisk N. Community pharmacists' perspectives on implementation of Medicines Use Review in Slovenia. Int J Clin Pharm. 2018.

8. Mantzourani E, Way CM, Hodson KL. Does an integrated information technology system provide support for community pharmacists undertaking Discharge Medicines Reviews? An exploratory study. Integr Pharm Res Pract. 2017;6:145-156..

9. Pisk N, Makovec UN, Kos M. Implementation of medicines use review in Slovenia: Pharmacist's perspective. Int J Clin Pharm. 2017;39(1):246.

10. Elayeh E, Akour A, Almadaeen S, AlQhewii T, Basheti IA. Practice of pharmaceutical care in community pharmacies in Jordan. Trop J Pharm Res. 2017;16(2):463-470.

11. Smith M, McConnell B. Aligning community-based comprehensive medication management services with value-based payment reform. J Am Pharm Assoc. 2017;57(3).

12. Donyai P, Hindi A, Patel N, Parkhurst C. A nationwide questionnaire study of patient satisfaction with the Medicines Use Review pharmacy service. Int J Pharm Pract. 2017;25:6-7.

13. Eze UI, Onasanya O. Evaluation of awareness, benefits and willingness to pay for pharmaceutical care (PC) services among older adults. Value Heal. 2017;20(5):A365. 
14. Neyarapally GA, Smith MA. Variability in state Medicaid medication management initiatives. Res Social Adm Pharm. 2017;13(1):214-223.

15. Jorgenson D, Currie K, Evans C, Mansell K, Perepelkin J. Community pharmacists' experiences with the Saskatchewan Medication Assessment Program. Can Pharm J. 2017;150(4):S23.

16. Rutter P, Ramsbottom H, Fitzpatrick R. Community pharmacist perceptions of delivering post-hospital discharge Medicines Use Reviews for elderly patients. Int J Clin Pharm. 2017;39(1):33-36.

17. MacKeigan LD, ljaz N, Bojarski EA, Dolovich L. Implementation of a reimbursed medication review program: Corporate and pharmacy level strategies. Res Social Adm Pharm. 2017;13(5):947-958.

18. Smith MA, Spiggle S, McConnell B. Strategies for community-based medication management services in value-based health plans. Res Social Adm Pharm. 2017;13(1):48-62.

19. Herrera H, Patel S. Community pharmacy and the delivery of Medicines Use Reviews and Prescription Intervention services - Are we enhancing patient care or meeting targets? Int J Pharm Pract. 2017;25:20-21.

20. Aimaurai S, Jumpated A, Krass I, Dhippayom T. Patient opinions on medicine-use review: exploring an expanding role of community pharmacists. Patient Prefer Adherence. 2017;11:751760.

21. Guthrie KD, Stoner SC, Hartwig DM, et al. Physicians' Preferences for Communication of Pharmacist-Provided Medication Therapy Management in Community Pharmacy. J Pharm Pract. 2017;30(1):17-24.

22. Hermansyah A, Sainsbury E, Krass I. The operation of a Research and Development (R\&D) program and its significance for practice change in community pharmacy. PLoS One. 2017;12(9):e0184954.

23. Lakic D, Stevic I, Odalovic M, Vezmar-Kovacevic S, Tadic I. Patients' willingness to pay for cognitive pharmacist services in community pharmacies. Croat Med J. 2017;58(5):364-371..

24. Tomaszewski D, Cernohous T, Vaidyanathan R. Evaluating Patient Preferences for Different Incentive Programs to Optimize Pharmacist-Provided Patient Care Program Enrollment. J Manag care Spec Pharm. 2017;23(11):1140-1147.

25. Costa FA, Scullin C, Al-Taani G, et al. Provision of pharmaceutical care by community pharmacists across Europe: Is it developing and spreading? J Eval Clin Pract. 2017;23(6):13361347. 
26. Dosea AS, Brito GC, Santos LMC, et al. Establishment, Implementation, and Consolidation of Clinical Pharmacy Services in Community Pharmacies: Perceptions of a Group of Pharmacists. Qual Health Res. 2017;27(3):363-373. doi:10.1177/1049732315614294.

27. Kho BP, Hassali MA, Lim CJ, Saleem F. A qualitative study exploring professional pharmacy services offered by community pharmacies in the state of Sarawak, Malaysia. J Pharm Heal Serv Res. 2017;8(3):201-208. http://onlinelibrary.wiley.com/journal/10.1111/(ISSN)1759-8893

28. Schulte N, Ruisinger JF, Prohaska ES, Steele KM, Melton BL. Comprehensive medication review recipients' opinions, actions, and information recall. J Am Pharm Assoc (2003). 2017;57(3):407-411.

29. Pedro ML, Mir , a I, Da Costa FA. Barriers and facilitators for the implementation of advanced pharmaceutical services. Int J Clin Pharm. 2016;38(4):1007.

30. Jacobs S, Fegan T, Bradley F, et al. Pharmacists' views of the extraorganisational factors affecting clinical productivity in english community pharmacies. Int J Pharm Pract. 2016;24:1718.

31. Guirguis L, Johnson S. Mind the gap: Patient and pharmacist engagement in community pharmacy. Int J Pharm Pract. 2016;24:14.

32. Volmer D, Ventmann I, Raal A, Airaksinen M. Public perception about community pharmacies and community pharmacy services 1993-2015 in Tartu, Estonia. Int J Pharm Pract. 2016;24:57.

33. Mantzourani E, Ellis L, Parry-Jones G, Hodson K, Way C. Does an integrated information technology system provide support for community pharmacists undertaking discharge medicines reviews? Int J Pharm Pract. 2016;24:82-83.

34. Rosenthal M, Tsao NW, Tsuyuki RT, Marra CA. Identifying relationships between the professional culture of pharmacy, pharmacists' personality traits, and the provision of advanced pharmacy services. Res Social Adm Pharm. 2016;12(1):56-67.

35. Smith M, Brown P, Shea C, Ferreri S. Barriers to providing comprehensive medication reviews in community pharmacies. J Am Pharm Assoc. 2016;56(3):e67-e68.

36. Latif A, Waring J, Watmough D, et al. Examination of England's New Medicine Service (NMS) of complex health care interventions in community pharmacy. Res Social Adm Pharm. 2016;12(6):966-989.

37. Ung COL, Chao CK, Hu Y, et al. Community pharmacists' understanding, attitudes, practice and perceived barriers related to providing pharmaceutical care: A questionnaire-based survey in Macao. Trop J Pharm Res. 2016;15(4):847-854. 
38. Rodgers RM, Gammie SM, Loo RL, Corlett SA, Krska J. Comparison of pharmacist and public views and experiences of community pharmacy medicines-related services in England. Patient Prefer Adherence. 2016;10:1749-1758..

39. Deepalakshmi M, Devipriya T, Arun KP, Ponnusankar S. Knowledge, attitude and practice of community pharmacists towards cognitive pharmaceutical care services in Tamil Nadu, India. Indian J Pharm Sci. 2016;78(6):844-849.

40. Kangwol P, Anantachoti P. Factors affecting community pharmacists' intention to provide medication management program and disease screening in Bangkok. Thai J Pharm Sci. 2016;40(3):158-166.

41. Pestka DL, Frail CK, Palombi LC, Von Hoff BA, Conway JM, Sorensen TD. Strategies and steps fostering the success of medication management services in community pharmacies. J Am Pharm Assoc. 2016;56(5):504-512.e1. doi:10.1016/j.japh.2016.05.001.

42. Mobley-Bukstein W, Ulrich EE, Tenhouse A. Quantifying the relationship between medication management and patient interest in MTM service. J Pharm Heal Serv Res. 2016;7(4):263-268. http://onlinelibrary.wiley.com/journal/10.1111/(ISSN)1759-8893

43. Farris KB, Salgado TM, Aneese N, et al. Effect of Clinical and Attitudinal Characteristics on Obtaining Comprehensive Medication Reviews. J Manag care Spec Pharm. 2016;22(4):388-395.

44. Merks P, Swieczkowski D, Jaguszewski MJ. Patients' perception of pharmaceutical services available in a community pharmacy among patients living in a rural area of the United Kingdom. Pharm Pract (Granada). 2016;14(3):774..

45. Anthony G, MacLure K, Cunningham S, et al. A qualitative exploration of pharmacist provision of pharmaceutical care services to patients in dispensing medical practices in remote and rural settings of Scotland. Int J Pharm Pract. 2016;24:5.

46. Dymek J, Skowron A, Golda A, Polak W, Pelka P, Molenda A. Medication review: What pharmacists can do in Poland. Int J Clin Pharm. 2016;38(4):1010..

47. Casserlie LM, Dipietro Mager NA. Pharmacists' perceptions of advancing public health priorities through medication therapy management. Pharm Pract. 2016;14(3):1-6.

48. Hasan S, Stewart K, Chapman CB, Hasan MY, Kong DCM. Physicians' attitudes towards provision of primary care services in community pharmacy in the United Arab Emirates. Int J Pharm Pract. 2015;23(4):274-282.

49. Tsao NW, Khakban A, Gastonguay L, Li K, Lynd LD, Marra CA. Perceptions of British Columbia residents and their willingness to pay for medication management services provided by pharmacists. Can Pharm J (Ott). 2015;148(5):263-273. 
50. Lucas B, Blenkinsopp A. Community pharmacists' experience and perceptions of the New Medicines Service (NMS). Int J Pharm Pract. 2015;23(6):399-406.

51. Huet AL, Frail CK, Lake LM, Snyder ME. Impact of passive and active promotional strategies on patient acceptance of medication therapy management services. J Am Pharm Assoc (2003). 2015;55(2):178-181.

52. Bardet JD, Vo TH, Bosson JL, Bedouch P, Allenet B. Patients', physicians' and pharmacists' viewpoints on the implementation of medication reviews in the French primary care. Int J Clin Pharm. 2015;37(1):199.

53. Waszyk-Nowaczyk M, Nowak M, Michalak M, Simon M. Physicians' Valuation of Individual Medication Management System (Imms) Implementation in Poznan (Poland) Community Pharmacies. Acta Pol Pharm. 2015;72(5):1039-1044.

54. Vella M, Grima M, Wirth F, et al. Consumer perception of community pharmacist extended professional services. J Pharm Heal Serv Res. 2015;6(2):91-96.

55. Krska J, Corlett SA, Gammie S, Loo RL, Rodgers RM. Medicine-related services: Do pharmacists see things the same way as the public? Int J Pharm Pract. 2015;23:25.

56. Mohamed SSE, Mahmoud AA, Ali AA. Sudanese community pharmacy practice and its readiness for change to patient care. Int J Pharm Pract. 2015;23(4):266-273.

57. Orrell C, Leong A, Boyd M, Boardman H. An evaluation of pharmacy customer views of services that should be available from community pharmacy. Int J Pharm Pract. 2015;23:59-60. 925.

58. Mamen A V, Hakonsen H, Kjome RLS, Gustavsen-Krabbesund B, Toverud EL. Norwegian elderly patients' need for drug information and attitudes towards medication use reviews in community pharmacies. Int J Pharm Pract. 2015;23(6):423-428.

59. Bishop AC, Boyle TA, Morrison B, et al. Public perceptions of pharmacist expanded scope of practice services in Nova Scotia. Can Pharm J (Ott). 2015;148(5):274-283.

60. Hessemer S, Schmid K, Schoeffski O. Willing to provide medication analysis? Predicting pharmacists' intention to provide medication analysis in community pharmacies in Germany. Value Heal. 2015;18(7):A544-A545.

61. Corlett SA, Michael D, Bhurawala MA, Dodds LJ. Discharge medicines use reviews (dMURs): Should community pharmacists be trying harder to identify patients who could benefit? Int J Pharm Pract. 2015;23:35.

62. Snyder ME, Earl TR, Gilchrist S, et al. Collaborative drug therapy management: case studies of three community-based models of care. Prev Chronic Dis. 2015;12:E39. 
63. Al-Nagar A, Desborough J. A feasibility study to investigate community pharmacy consultations. Int J Pharm Pract. 2014;22:31-32.

64. Avena-Woods C, Mantione M. Utilization of phar-macy interns and auxiliary staff in providing medication therapy management services. J Am Pharm Assoc. 2014;54(2):e146-e147.

65. Corlett S, Goel P, Kothari S, Dodds L. Are sufficient efforts being made by hospital pharmacy teams to encourage patients to access a Medicines Use review after discharge? Int J Pharm Pract. 2014;22:8-9.

66. Smith M, Cannon-Brel , L. , M, Spiggle S. Consumer, physician, and payer perspectives on primary care medication management services with a shared resource pharmacists network. Res Social Adm Pharm. 2014;10(3):539-553.

67. Waszyk-Nowaczyk M, Lawicki S, Michalak M, Simon M. Individual medication management system (IMMS) implementation in pharmacists' opinion. Acta Pol Pharm. 2014;71(3):509-514.

68. Babiker AH, Carson L, Awaisu A. Medication use review in Qatar: Are community pharmacists prepared for the extended professional role? Int J Clin Pharm. 2014;36(6):12411250. doi:10.1007/s11096-014-0025-8.

69. Latifa A, Watmough D, Salema NE, Elliott RA, Boyd MJ, Waring J. The New Medicines Service (NMS): Understanding its delivery in everyday practice. Int J Pharm Pract. 2014;22:67.

70. Zolezzi M, Bye L, Harrison J, Tsuyuki RT, Shaw JP. Provision of health disease screening and medication monitoring/management in New Zealand community pharmacies. J Pharm Pract Res. 2014;44(4):188-194.

71. Corlett SA, Dodds LJ, Gammie S. Do pharmacists think that medicine advisory services improve patient adherence? Int J Pharm Pract. 2014;22:34-35.

72. Wells KM, Thornley T, Boyd MJ, Boardman HF. Views and experiences of community pharmacists and superintendent pharmacists regarding the New Medicine Service in England prior to implementation. Res Soc Adm Pharm. 2014;10(1):58-71.

doi:10.1016/j.sapharm.2013.03.003.

73. Umaru N, Aslanpour Z, Adegbesan A, Bhogal N, Hussain Z, Geeson C. Medicines optimisation in older patients: A qualitative study of the views of hospital pharmacists on the Medicines Use Review (MUR) and New Medicines Service (NMS). Int J Pharm Pract. 2014;22:62.

74. Khan F, Lauder D, Hodson K. Signposting of patients discharged from hospital to community pharmacists to share discharge medication summaries with a view to receiving MUR or NMS. Int J Pharm Pract. 2014;22:104-105. 
75. Babiker A, Awaisu A, Carson L. Medication use review service in Qatar: A nationwide study on knowledge, attitudes and practices of community pharmacists. Int J Clin Pharm. 2014;36(4):852-853.

76. Arya V, Pinto S, Singer J, Khan T. Understanding awareness of pharmacist-led medication therapy management among primary care physicians in New York City using qualitative methods: part I. J Med Pract Manage. 2013;29(2):84-88.

77. Latif A, Boardman HF, Pollock K. Understanding the patient perspective of the English community pharmacy Medicines Use Review (MUR). Res Social Adm Pharm. 2013;9(6):949-957.

78. McMillan S, Sav A, Kelly F, King M, Whitty J, Wheeler A. Australian community pharmacy: Determinants influencing pharmacy choice. Int J Pharm Pract. 2013;21:34-35.

79. Hatah E, Braund R, Duffull SB, Tordoff J. General practitioners' views of pharmacists' current and potential contributions to medication review and prescribing in New Zealand. J Prim Health Care. 2013;5(3):223-233.

80. Rodgers R, Loo RL, Krska J. Public perception of medicines-related advisory services provided by pharmacies. Int J Pharm Pract. 2013;21:28-29.

81. Corlett SA, Dodds LJ, Rodgers RM. As pharmacists expand their clinical roles do the general public recognise what they can offer? Pharmacoepidemiol Drug Saf. 2013;22(6):672-673.

82. Hasan S, Sulieman H, Stewart K, Chapman CB, Hasan MY, Kong DCM. Assessing patient satisfaction with community pharmacy in the UAE using a newly-validated tool. Res Social Adm Pharm. 2013;9(6):841-850.

83. Latif A, Boardman HF, Pollock K. A qualitative study exploring the impact and consequence of the medicines use review service on pharmacy support-staff. Pharm Pract (Granada). 2013;11(2):118-124..

84. Thomas $\mathrm{CL}$, Allison M. Evaluation of a community pharmacy falls and fracture prevention medicine use review (MUR) service. Int J Pharm Pract. 2013;21:15-16.

85. Bhatti N, Devlin L, Farooq H, et al. Community pharmacists' experiences of managing patients' medicines after discharge from hospital: A preliminary study of discharge medicines use reviews (DMURs). Int J Pharm Pract. 2013;21:86-87.

86. Al-Nagar A, Skinner J, Salter C, Desborough J. Is building confidence in consultation skills the key to delivering more patient facing services in community pharmacy? Int J Pharm Pract. 2013;21:89-90.

87. Arya V, Pinto S, Singer J. Understanding awareness of pharmacist-led medication therapy management among primary care physicians in New York City, Part II: Findings and implications. J Med Pract Manage. 2013;29(3):187-194.. 
88. Doucette WR, Zhang Y, Chrischilles EA, et al. Factors affecting Medicare Part D beneficiaries' decision to receive comprehensive medication reviews. J Am Pharm Assoc (2003). 2013;53(5):482-487.

89. Duke M. Patient attitudes and expectations of pharmacist-provided clinical services in clinicbased community pharmacies. J Am Pharm Assoc. 2013;53(2):e73.

90. Latif A, Pollock K, Boardman HF. Medicines use reviews: a potential resource or lost opportunity for general practice? BMC Fam Pract. 2013;14:57.

91. Sheridan J, Butler R, Brandt T, Harrison J, Jensen M, Shaw J. Patients' and pharmacists' perceptions of a pilot Medicines Use Review service in Auckland, New Zealand. J Pharm Heal Serv Res. 2012;3(1):35-40.

92. Latif A, Pollock K, Boardman H. Medicines use reviews (MURs) in practice: Implementation and outcomes for patient care. Int J Clin Pharm. 2012;34(1):173-174.

93. Boyd MJ, Wells KM, Thornley T, Boardman HF. Views of community pharmacists regarding the New Medicine Service prior to its implementation. Int J Pharm Pract. 2012;20:22.

94. Hatah E, Braund R, Duffull S, Tordoff J. General practitioners' perceptions of pharmacists' new services in New Zealand. Int J Clin Pharm. 2012;34(2):364-373.

95. Kaae S, Christensen ST. Exploring long term implementation of cognitive services in community pharmacies - a qualitative study. Pharm Pract (Granada). 2012;10(3):151-158.

96. Tordoff J, Chang SY, Norris PT. Community pharmacists' perceptions of services that benefit older people in New Zealand. Int J Clin Pharm. 2012;34(2):342-350.

97. Wells KM, Thornley T, Boardman HF, Boyd MJ. Views of superintendent pharmacists regarding the New Medicine Service prior to its implementation. Int J Pharm Pract. 2012;20:2526.

98. Dewsbury C, Acharya S, Khinda R, Singh P. Public use and views on community pharmacy public health services in England. Int J Pharm Pract. 2012;20:38.

99. Lauffenburger JC, Vu MB, Burkhart J, Weinberger M, Roth MT. Design of a medication therapy management program for Medicare beneficiaries: qualitative findings from patients and physicians. Am J Geriatr Pharmacother. 2012;10(2):129-138.

100. Chui MA, Mott DA, Maxwell L. A qualitative assessment of a community pharmacy cognitive pharmaceutical services program, using a work system approach. Res Social Adm Pharm. 2012;8(3):206-216.

101. Latif A, Pollock K, Boardman HF. The contribution of the Medicines Use Review (MUR) consultation to counseling practice in community pharmacies. Patient Educ Couns. 2011;83(3):336-344. 
102. Latif A, Boardman H, Pollock K. Exploring community pharmacy medicines use reviews as a means of improving the clinical and cost effectiveness of prescribed medicines and reducing waste. Pharmacoepidemiol Drug Saf. 2011;20(6):666. 963.

103. Boardman H, Latif A, Pollock K. Impact and consequences of Medicines Use Reviews (MURs) on the workload in community pharmacy. Int J Pharm Pract. 2011;19:32-33.

104. Joshi N, West D, Banahan B, Bentley J, Bloodworth L, Ross L. Preliminary evaluation of a MTM program on patient-reported outcomes in the Mississippi Delta. J Am Pharm Assoc. 2011;51(2):264.

105. Deslandes R, Frazer H. An exploration of the views of general practitioners on the role of the community pharmacist. Int J Pharm Pract. 2011;19:11.

106. Waterfield J, Dhir A. An exploration of the views of community pharmacists on the relationship between Medicines Use Reviews (MURs) and public health. Int J Pharm Pract. 2011;19:68-69.

107. Kak K, Agro P, Agro K, Folia C, Lillie E. MedsChecks: Are community pharmacists willing to bring in external assistance? Can Pharm J. 2011;144(5):e20.

108. Coover K, Tilleman J, Begley K, et al. Evaluation of participation in medication therapy management services by pharmacy students on advanced practice rotations. J Am Pharm Assoc. 2011;51(2):259-260.

109. Blenkinsopp A, Savage I, Bennett M, Closs J. Improving the management of cancer pain in the community: Is there a place for MURs? Int J Pharm Pract. 2011;19:91-92.

110. Young S, Phillips L, Clark D, Kelly D. Determining public attitudes and interest in expanded pharmacist services. Can Pharm J. 2011;144(5):e57.

111. Hong SH, Liu J, Wang J, Brown L, White-Means S. Conjoint analysis of patient preferences on Medicare medication therapy management. J Am Pharm Assoc (2003). 2011;51(3):378-387.

112. Osborne MA, Snyder ME, Hall DL, Coley KC, McGivney MS. Evaluating Pennsylvania Pharmacists' Provision of Community-based Patient Care Services to Develop a Statewide Practice Network. Inov Pharm. 2011;2(4):61.

113. Latif A, Boardman $\mathrm{H}$, Pollock K. Reasons involved in selecting patients for a Medicines Use Review (MUR): Exploring pharmacist and staff choices. Int J Pharm Pract. 2011;19:31-32.

114. Kaae S, Sorensen EW, Norgaard LS. Exploring communications around medication review in community pharmacy. Int J Clin Pharm. 2011;33(3):529-536.

115. Shah B, Chawla S. A needs assessment for development and provision of medication therapy management services in New York City. J Pharm Pract. 2011;24(3):339-344. 
116. Pojskic N, Mackeigan L, Boon H, Ellison P, Breslin C. Ontario family physician readiness to collaborate with community pharmacists on drug therapy management. Res Social Adm Pharm. 2011;7(1):39-50.

117. Latif A, Boardman H, Pollock K. A critical look at the Medicines Use Review consultation as a means of collaborating with patients about their medicines. Int J Pharm Pract. 2011;19:25.

118. Blake KB, Madhavan SS. Perceived barriers to provision of medication therapy management services (MTMS) and the likelihood of a pharmacist to work in a pharmacy that provides MTMS. Ann Pharmacother. 2010;44(3):424-431.

119. McGrath SH, Snyder ME, Duenas GG, Pringle JL, Smith RB, McGivney MS. Physician perceptions of pharmacist-provided medication therapy management: qualitative analysis. J Am Pharm Assoc (2003). 2010;50(1):67-71.

120. Latif A, Pollock K, Boardman H. Why do patients accept or decline the invitation for a Medicines Use Review? Int J Pharm Pract. 2010;18:21.

121. Chui M, Mott D, Maxwell L, Kreling D, Martin B. Qualitative assessment of important pharmacy work system facilitators to pharmacist role expansion. J Am Pharm Assoc. 2010;50(2):259.

122. Khan S, Avena-Woods C. Medicare Part D in community pharmacy: Continued impact and related issues. Pharmacotherapy. 2010;30(10):377e-378e.

123. Iqbal S, Wood K. Exploring patient opinions of MURs. Int J Pharm Pract. 2010;18:20.

124. Feletto E, Wilson LK, Roberts AS, Benrimoj SI. Building capacity to implement cognitive pharmaceutical services: Quantifying the needs of community pharmacies. Res Soc Adm Pharm. 2010;6(3):163-173. doi:10.1016/j.sapharm.2009.08.003.

125. Cowley J, Gidman W, McGregor L, Andoh N. Exploring community pharmacists' experience and opinions of Medication Review services in England, Wales and Scotland. Int J Pharm Pract. 2010;18:88-89.

126. Latif A, Mahmood K, Boardman H. Medicines Use Reviews - How have pharmacists' views changed? Int J Pharm Pract. 2010;18:68-69. 979.

127. Niquille A, Lattmann C, Bugnon O. Medication reviews led by community pharmacists in Switzerland: a qualitative survey to evaluate barriers and facilitators. Pharm Pract (Granada). 2010;8(1):35-42..

128. Latif A, Boardman H, Pollock K. Exploring "ad hoc" medicines use reviews: Methodological and ethical considerations. Int J Pharm Pract. 2010;18:40.

129. Bryant L, Coster G, McCormick R. Community pharmacist perceptions of clinical medication reviews. J Prim Health Care. 2010;2(3):234-242. 
130. McDonald R, Cheraghi-Sohi S, S, ers C, Ashcroft D. Professional status in a changing world: The case of medicines use reviews in English community pharmacy. Soc Sci Med. 2010;71(3):451-458.

131. van den Berg M, Donyai P. How was patient empowerment portrayed in information leaflets describing the community pharmacy Medicines Use Review service in the UK? Patient Educ Couns. 2010;80(2):274-276.

132. Friedrich M, Zgarrick D, Masood A, Montuoro J. Patients' needs and interests in a self-pay medication therapy management service. J Am Pharm Assoc (2003). 2010;50(1):72-77.

133. Harding G, Wilcock M. Community pharmacists' perceptions of medicines use reviews and quality assurance by peer review. Pharm World Sci. 2010;32(3):381-385.

134. Jee S, Schafheutle E, Hassell K, Noyce P. Appraisal systems within community pharmacy: Will they prove useful for revalidation? Int J Pharm Pract. 2010;18:97.

135. Moczygemba LR, Barner JC, Brown CM, et al. Patient satisfaction with a pharmacistprovided telephone medication therapy management program. Res Social Adm Pharm. 2010;6(2):143-154.

136. Feletto E, Wilson LK, Roberts AS, Benrimoj SI. Flexibility in community pharmacy: a qualitative study of business models and cognitive services. Pharm World Sci. 2010;32(2):130138.

137. Blake KB, Madhavan SS, Scott VG, Meredith Elswick BL. Medication therapy management services in West Virginia: pharmacists' perceptions of educational and training needs. Res Social Adm Pharm. 2009;5(2):182-188.

138. Patel P, Lefteri K. The perceived value of MURs to patients in a rural community. Int J Pharm Pract. 2009;17:B79-B80.

139. James D, Birch V, John DN. Pharmacists' views about the medicines use review Accreditation process. Int J Pharm Pract. 2009;17:B89-B90.

140. Law A V, Okamoto MP, Brock K. Ready, willing, and able to provide MTM services?: A survey of community pharmacists in the USA. Res Social Adm Pharm. 2009;5(4):376-381.

141. MacIntosh C, Weiser C, Wassimi A, et al. Attitudes toward and factors affecting implementation of medication therapy management services by community pharmacists. J Am Pharm Assoc (2003). 2009;49(1):26-30.

142. Lounsbery JL, Green CG, Bennett MS, Pedersen CA. Evaluation of pharmacists' barriers to the implementation of medication therapy management services. J Am Pharm Assoc (2003). 2009;49(1):51-58. 
143. Khideja N. Community pharmacists' experience and perceptions of Medicines Use Review: initial and ongoing training of community pharmacists in the black country region - West Midlands. Int J Pharm Pract. 2009;17:B60-B61.

144. Truong HA, Layson-Wolf C, de Bittner MR, Owen JA, Haupt S. Perceptions of patients on Medicare Part D medication therapy management services. J Am Pharm Assoc (2003). 2009;49(3):392-398.

145. De Amezua CMJ, Martinez-Martinez F, Gastelurrutia MA. Opinions of pharmacists, experts in delivering advanced Cognitive Services such as Medicine management, about the lack of implementation of These Services in spain. Pharm World Sci. 2009;31(4):500.

146. Alkhateeb FM, Clauson KA, McCafferty R, Latif DA. Physician attitudes toward pharmacist provision of medication therapy management services. Pharm World Sci. 2009;31(4):487-493.

147. Kuhn CH, Casper KA, Green TR. Assessing Ohio grocery store patrons' perceptions of a comprehensive medication review. J Am Pharm Assoc (2003). 2009;49(6):787-791.

148. Gadkari AS, Mott DA, Kreling DH, Bonnarens JK. Pharmacy characteristics associated with the provision of drug therapy services in nonmetropolitan community pharmacies. J Rural Health. 2009;25(3):290-295.

149. Gastelurrutia MA, Benrimoj SIC, Castrillon CC, et al. Facilitators for practice change in Spanish community pharmacy. Pharm World Sci. 2009;31(1):32-39.

150. Bradley CT, Henman MC. The view of key stakeholders regarding the role of community pharmacists. Pharm World Sci. 2009;31(4):506.

151. Alkhateeb FM, Unni E, Latif D, Shawaqfeh MS, Al-Rousan RM. Physician attitudes toward collaborative agreements with pharmacists and their expectations of community pharmacists' responsibilities in West Virginia. J Am Pharm Assoc (2003). 2009;49(6):797-800.

152. Bryant LJM, Coster G, Gamble GD, McCormick RN. General practitioners' and pharmacists' perceptions of the role of community pharmacists in delivering clinical services. Res Social Adm Pharm. 2009;5(4):347-362.

153. Castrillon CC, Faus MJ, Gastelurrutia MA. Patients' perceptions over a generalized medicine management service, in a Spanish community pharmacy. Pharm World Sci. 2009;31(4):499.

154. Bright DR, Lengel AJ, Powers MF. Pharmacists' perceptions of barriers to implementing medication therapy management and the role of pharmacy technicians in overcoming the barriers. J Pharm Technol. 2009;25(6):361-367. 
155. Millonig MK. Mapping the route to medication therapy management documentation and billing standardization and interoperabilility within the health care system: meeting proceedings. J Am Pharm Assoc (2003). 2009;49(3):372-382.

156. Lee E, Braund R, Tordoff J. Examining the first year of Medicines Use Review services provided by pharmacists in New Zealand: 2008. N Z Med J. 2009;122(1293):3566.

157. Schommer JC, Planas LG, Johnson KA, Doucette WR. Pharmacist-provided medication therapy management (part 1): provider perspectives in 2007. J Am Pharm Assoc (2003). 2008;48(3):354-363.

158. Schommer JC, Planas LG, Johnson KA, Doucette WR. Pharmacist-provided medication therapy management (part 2): Payer perspectives in 2007. J Am Pharm Assoc (2003). 2008;48(4):478-486.

159. Wilcock M, Harding G. What do pharmacists think of MURs and do they change prescribed medication? Pharm J. 2008;281(7514):163-167.

160. Harding G, Wilcock M. What do pharmacists think of peer review of medicine use reviews? Pharm J. 2008;281(7531):674-676.

161. Moczygemba LR, Barner JC, Roberson K. Texas pharmacists' opinions about and plans for provision of medication therapy management services. J Am Pharm Assoc (2003). 2008;48(1):38-45.

162. Law A V, Okamoto MP, Brock K. Perceptions of Medicare Part D enrollees about pharmacists and their role as providers of medication therapy management. J Am Pharm Assoc (2003). 2008;48(5):648-653.

163. Bissell P, Blenkinsopp A, Short D, Mason L. Patients' experiences of a community pharmacy-led medicines management service. Health Soc Care Community. 2008;16(4):363369.

164. Franic DM, Tucker LT, Haddock SM. Pharmaceutical care: What's in a name? A qualitative analysis of patient perceptions of pharmacy practice. J Pharm Technol. 2008;24(4):191-201.

165. Roberts AS, Benrimoj SI, Chen TF, Williams KA, Aslani P. Practice change in community pharmacy: quantification of facilitators. Ann Pharmacother. 2008;42(6):861-868.

166. Urban R, Rivers P, Morgan J. Perceptions of medicines use reviews - The views of community pharmacists within a West Yorkshire primary care trust. Pharm J. 2008;281(7519):303-305.

167. Bradley F, Wagner AC, Elvey R, Noyce PR, Ashcroft DM. Determinants of the uptake of medicines use reviews (MURs) by community pharmacies in England: a multi-method study. Health Policy. 2008;88(2):258-268. 
168. Latif A, Boardman H. Community pharmacists' attitudes towards medicines use reviews and factors affecting the numbers performed. Pharm World Sci. 2008;30(5):536-543.

169. Schuh MJ, Droege M. Cognitive services provided by pharmacists: is the public willing to pay for them? Consult Pharm. 2008;23(3):223-230.

170. Mark MP. The general pharmacy work explored in The Netherlands. Pharm World Sci. 2008;30(4):353-359.

171. Gastelurrutia MA, Fern , ez-Llimos F, Benrimoj SI, Castrillon CC, Faus MJ. Barriers for the implementation of cognitive services in Spanish community pharmacies. Aten Primaria. 2007;39(9):465-470.

172. Montgomery AT, Kalvemark-Sporrong S, Henning M, Tully MP, Kettis-Lindblad A. Implementation of a pharmaceutical care service: prescriptionists', pharmacists' and doctors' views. Pharm World Sci. 2007;29(6):593-602.

173. Laaksonen R, Bates I, Duggan C. Training, clinical medication review performance and selfassessed competence: Investigating influences. Pharm Educ. 2007;7(3):257-265.

174. Tinelli M, Bond C, Blenkinsopp A, et al. Patient evaluation of a community pharmacy medications management service. Ann Pharmacother. 2007;41(12):1962-1970.

175. Laaksonen R, Mills ER, Duggan C, Davies JG, Bates I, Mackie CA. The effect of training and service provision on the self-assessed competence of community pharmacists. Int J Pharm Pract. 2007;15(2):141-147.

176. Wilcock M, Harding G. General practitioners' perceptions of medicines use reviews by pharmacists. Pharm J. 2007;279(7476):501-503.

177. Scott A, Bond C, Inch J, Grant A. Preferences of community pharmacists for extended roles in primary care: a survey and discrete choice experiment. Pharmacoeconomics. 2007;25(9):783792.

178. Hill P, Dowse R. Cognitive pharmaceutical services and the community pharmacist: Are south african patients receiving them and are they willing to pay? Int J Pharm Pract. 2007;15(3):201-208.

179. Hansen RA, Roth MT, Brouwer ES, Herndon S, Christensen DB. Medication therapy management services in North Carolina community pharmacies: current practice patterns and projected demand. J Am Pharm Assoc (2003). 2006;46(6):700-706.

180. Padiyara RS, Rabi SM. Physician perceptions of pharmacist provision of outpatient medication therapy management services. J Am Pharm Assoc (2003). 2006;46(6):660-663. 
181. Kreling DH, Doucette WR, Mott DA, Gaither CA, Pedersen CA, Schommer JC. Community pharmacists' work environments: Evidence from the 2004 National Pharmacist Workforce Study. J Am Pharm Assoc. 2006;46(3):331-339.

182. Herbert KE, Urmie JM, Newl , A. , B, Farris KB. Prediction of pharmacist intention to provide Medicare medication therapy management services using the theory of planned behavior. Res Social Adm Pharm. 2006;2(3):299-314.

183. Hopp TR, Klinke BO, Sorensen EW, Herborg H, Roberts AS. Implementation of cognitive pharmaceutical services in Danish community pharmacies - Perceptions of strategists and practitioners. Int J Pharm Pract. 2006;14(1):37-49.

184. Doucette WR, Kreling DH, Schommer JC, Gaither CA, Mott DA, Pedersen CA. Evaluation of community pharmacy service mix: evidence from the 2004 National Pharmacist Workforce Study. J Am Pharm Assoc (2003). 2006;46(3):348-355.

185. Schommer JC, Pedersen CA, Worley MM, et al. Provision of risk management and risk assessment information: the role of the pharmacist. Res Social Adm Pharm. 2006;2(4):458-478.

186. Brock KA, Casper KA, Green TR, Pedersen CA. Documentation of patient care services in a community pharmacy setting. J Am Pharm Assoc. 2006;46(3):378-384.

187. Schommer JC, Pedersen CA, Gaither CA, Doucette WR, Kreling DH, Mott DA. Pharmacists' desired and actual times in work activities: evidence of gaps from the 2004 National Pharmacist Workforce Study. J Am Pharm Assoc (2003). 2006;46(3):340-347. 5.

188. Boyd ST, Boyd LC, Zillich AJ. Medication therapy management survey of the prescription drug plans. J Am Pharm Assoc (2003). 2006;46(6):692-699.

189. Hopp TR, Sorensen EW, Herborg H, Roberts AS. Implementation of cognitive pharmaceutical services (CPS) in professionally active pharmacies. Int J Pharm Pract. 2005;13(1):21-31.

190. Tootelian DH, Rolston LW, Negrete MJ. Consumer receptiveness to non-traditional roles for community pharmacists. Health Mark Q. 2005;23(1):43-56.

191. Roberts AS, Benrimoj SIC, Chen TF, Williams KA, Hopp TR, Aslani P. Understanding practice change in community pharmacy: a qualitative study in Australia. Res Social Adm Pharm. 2005;1(4):546-564.

192. Tweedie A, Rutter P, Jones I. Independent pharmacy contractors' views on the new medicines management service. Pharm J. 2004;273(7309):121-123.

193. Dinnie E, Bond C, Watson M. Community pharmacists' attitudes to medicines management: A qualitative study. Int J Pharm Pract. 2004;12(4):217-222. 
194. Roberts AS, Hopp T, Sorensen EW, et al. Understanding practice change in community pharmacy: a qualitative research instrument based on organisational theory. Pharm World Sci. 2003;25(5):227-234.

195. Bislew HD, Sorensen TD. Use of focus groups as a tool to enhance a pharmaceutical care practice. J Am Pharm Assoc (2003). 2003;43(3):424.

196. Hughes CM, McCann S. Perceived interprofessional barriers between community pharmacists and general practitioners: a qualitative assessment. Br J Gen Pract. 2003;53(493):600-606. 5.

197. Krska J, Veitch GBA. Providing pharmaceutical care - The views of Scottish pharmacists. Pharm J. 2001;267(7170):549-555. 47.

198. Odedina FT, Segal R, Hepler CD, Lipowski E, Kimberlin C. Changing pharmacists' practice pattern: Pharmacists' implementation of pharmaceutical care factors. J Soc Adm Pharm. 1996;13(2):74-88.

199. Odedina FT, Segal R, Hepler CD. Providing pharmaceutical care in community practice: Differences between providers and non-providers of pharmaceutical care. J Soc Adm Pharm. 1995;12(4):170-180.

200. Miller MJ, Ortmeier BG. Factors influencing the delivery of pharmacy services. Am Pharm. 1995;(1):39-45.

201. Raisch DW. Barriers to providing cognitive services. Am Pharm. 1993;(12):54-58.

202. Messerli M, Lottaz V, Vriends N, Hersberger KE. Collaborative development of outcome measures to investigate intermediate medication reviews provided in community pharmacies. Int J Clin Pharm. 35(3):502. 


\section{iii) Observational / Descriptive Studies}

1. Turner $\mathrm{K}$, Trogdon J, Weinberger $\mathrm{M}$, et al. Identifying pharmacy characteristics associated with effective implementation of a medicaid community pharmacy care management intervention. J Am Pharm Assoc. 2018;58(3):e79-e80.

2. Rosenthal MM, Holmes ER. The Professional Culture of Community Pharmacy and the Provision of MTM Services. Pharm (Basel, Switzerland). 2018;6(2).

3. Koyama T, Onoue $\mathrm{H}$, Ohshima A, et al. Trends in the medication reviews of community pharmacies in Japan: a nationwide retrospective study. Int J Clin Pharm. 2018;40(1):101-108.

4. Imfeld-Isenegger T, Costa FA, Soares IB, et al. PRACTISE-PharMacist-led CogniTive service in Europe (part B): Mapping of medication review services across Europe. Int J Clin Pharm. 2018;40(2):503.

5. Domiati S, Sacre H, Lahoud N, Sili G, Salameh P. Knowledge of and readiness for medication therapy management among community pharmacists in Lebanon. Int J Clin Pharm. 2018.

6. Alzubaidi H, Saidawi W, Mc Namara K. Pharmacist views and pharmacy capacity to deliver professional services in the United Arab Emirates. Int J Clin Pharm. 2018.

7. Alhaddad MS. Youth Experience With Community Pharmacy Services and Their Perceptions Toward Implementation of Medication Therapy Management Services by Community Pharmacists in the Western Region of Saudi Arabia. Ther Innov Regul Sci. 2018:2168479018769299.

8. Adeoye OA, Lake LM, Lourens SG, Morris RE, Snyder ME. What predicts medication therapy management completion rates? The role of community pharmacy staff characteristics and beliefs about medication therapy management. J Am Pharm Assoc (2003). 2018.

9. Thayer N, Willis S, Jacobs S. Targeted Medicines Use Review activity: Association with local health need. Int J Pharm Pract. 2017;25:21-22.

10. Stanway VE, El-Ghannam M, Murphy EM. Analysing uptake of new medicine service in English community pharmacies. Value Heal. 2017;20(9):A508.

11. Smith MG, Shea CM, Brown P, Wines K, Farley JF, Ferreri SP. Pharmacy characteristics associated with the provision of medication management services within an integrated care management program. J Am Pharm Assoc (2003). 2017;57(2):217-221.e211.

12. Seidling HM, Send AFJ, Bittmann J, et al. Medication review in German community pharmacies - Post-hoc analysis of documented drug-related problems and subsequent interventions in the ATHINA-project. Res Social Adm Pharm. 2017;13(6):1127-1134. 
13. Noain A, Garcia-Cardenas V, Gastelurrutia MA, et al. Cost analysis for the implementation of a medication review with follow-up service in Spain. Int J Clin Pharm. 2017;39(4):750-758.

14. Madjar B, Makovec UN, Kos M. Retrospective analysis of medicines use review in Slovenia. Int J Clin Pharm. 2017;39(1):245-246.

15. MacCallum L, Consiglio G, MacKeigan L, Dolovich L. Uptake of Community PharmacistDelivered MedsCheck Diabetes Medication Review Service in Ontario between 2010 and 2014. Can J diabetes. 2017;41(3):253-258.

16. Hann M, Schafheutle El, Bradley F, et al. Organisational and extraorganisational determinants of volume of service delivery by English community pharmacies: a cross-sectional survey and secondary data analysis. BMJ Open. 2017;7(10):e017843.

17. El-Ghannam M, Stanway VE, Murphy EM. Assessing uptake of medicine use reviews in English community pharmacies. Value Heal. 2017;20(9):A671.

18. Doucette WR, Rippe JJ, Gaither CA, Kreling DH, Mott DA, Schommer JC. Influences on the frequency and type of community pharmacy services. J Am Pharm Assoc (2003). 2017;57(1):7276.e71.

19. Deal HJ, Cooke CA, Ingram EML, Sketris IS. Adoption of the Nova Scotia (Canada) Community Pharmacy Medication Management Program, 1-Year Post-Initiation. J Popul Ther Clin Pharmacol. 2017;24(1):e46-e55.

20. Scott DM, Strand M, Undem T, Anderson G, Clarens A, Liu X. Assessment of pharmacists' delivery of public health services in rural and urban areas in lowa and North Dakota. Pharm Pract (Granada). 2016;14(4):836.

21. Pechlivanoglou P, Abrahamyan L, Mackeigan L, et al. Factors affecting the delivery of community pharmacist-led medication reviews: evidence from the MedsCheck annual service in Ontario. BMC Health Serv Res. 2016;16(1):666.

22. Greising C, Kossler K, Freyer J, et al. The status of the performance of medication reviews in German community pharmacies and assessment of the practical performance. Int J Clin Pharm. 2016;38(6):1425-1435.

23. Dolovich L, Consiglio G, MacKeigan L, et al. Uptake of the MedsCheck annual medication review service in Ontario community pharmacies between 2007 and 2013. Can Pharm J (Ott). 2016;149(5):293-302.

24. Pechlivanoglou P, Abrahamyan L, MacKeigan L, et al. Predictors of Ontario's medication review program (MedsCheck annual service) utilization among Ontario seniors. Pharmacoepidemiol Drug Saf. 2015;24:89-90. 
25. Jacobs S, Hann M, Wagner A, Schafheutle E. Medicines use review (MUR) activity in English community pharmacies: Associations with pharmacy type and population need. Int J Pharm Pract. 2015;23:48.

26. Jacobs S, Hann M, Elvey R, et al. Organisational factors associated with variation in dispensing and medicine use review (MUR) activity in a sample of English community pharmacies. Int J Pharm Pract. 2015;23:47.

27. Doucette W, Gaither C, Schommer J, et al. Pharmacy service mix reported in the 2014 national pharmacist workforce survey. J Am Pharm Assoc. 2015;55(2):e211-e212.

28. Kempen TGH, Van De Steeg-Van Gompel CHPA, Hoogland P, Liu Y, Bouvy ML. Large scale implementation of clinical medication reviews in Dutch community pharmacies: Drug-related problems and interventions. Int J Clin Pharm. 2014;36(3):630-635

29. Tucker $\mathrm{R}$. The medicines use review in patients with chronic skin diseases: are pharmacists doing them and how confident are they? Int J Pharm Pract. 2013;21(3):202-204.

30. Larson S, Drake S, Anderson L, Larson T. Adoption of medication therapy management programs in Minnesota: 2006-11. J Am Pharm Assoc (2003). 2013;53(3):254-260.

31. Hata M, Klotz R, Wang W. Examining the design and accessibility of medicare medication therapy management programs in California. J Am Pharm Assoc. 2012;52(2):241.

32. Doucette W, McDonough R, Mormann M, Cernetchi R, Urmie J. Analyses of sustainability of pharmacy services at an independent community pharmacy. J Am Pharm Assoc. 2012;52(2):251-252.

33. Doucette WR, McDonough RP, Mormann MM, Vaschevici R, Urmie JM, Patterson BJ. Threeyear financial analysis of pharmacy services at an independent community pharmacy. J Am Pharm Assoc (2003). 2012;52(2):181-187.

34. Beatty SJ, McCormick KM, Beale DJ, et al. Current trends in outpatient pharmacy services and billing. J Am Pharm Assoc (2003). 2012;52(2):154-160.

35. Rupp MT. Analyzing the costs to deliver medication therapy management services. J Am Pharm Assoc (2003). 2011;51(3):e19-e27.

36. MacAdam AB, Sherwood J. An investigation into the provision of Medicines Use Reviews (MURs) by community pharmacists within Brighton and Hove Primary Care Trust. Int J Pharm Pract. 2011;19:31.

37. Feletto E, Wilson LK, Roberts AS, Benrimoj SI. Measuring organizational flexibility in community pharmacy: Building the capacity to implement cognitive pharmaceutical services. Res Social Adm Pharm. 2011;7(1):27-38. 
38. Deal H, Borden N, Wilson JA, Cooke C, Gillis J. Findings from the Nova Scotia seniors medication review program. Can Pharm J. 2011;144(5):e49.

39. Brache E, O'Riordan G, Clemerson J. A peer review of Medicine Use Reviews (MURs) conducted by community pharmacists in Guernsey during the thirteen months after their inception. Int J Pharm Pract. 2011;19:66-67.

40. McDonough RP, Harthan AA, McLeese KE, Doucette WR. Retrospective financial analysis of medication therapy management services from the pharmacy's perspective. J Am Pharm Assoc (2003). 2010;50(1):62-66.

41. Haag JD, Stratton TP. Patient care services in rural Minnesota community pharmacies. J Am Pharm Assoc (2003). 2010;50(4):508-516.

42. Barnett MJ, Frank J, Wehring H, et al. Analysis of pharmacist-provided medication therapy management (MTM) services in community pharmacies over 7 years. J Manag Care Pharm. 2009;15(1):18-31.

43. Blenkinsopp A, Bond C, Celino G, Inch J, Gray N. Medicines use review: Adoption and spread of a service innovation. Int J Pharm Pract. 2008;16(4):271-276.

44. Brooks JM, Klepser DG, Urmie JM, Farris KB, Doucette WR. Effect of local competition on the willingness of community pharmacies to supply medication therapy management services. J Health Hum Serv Adm. 2007;30(1):4-27.

45. McLean WM, MacKeigan LD. When does pharmaceutical care impact health outcomes? A comparison of community pharmacy-based studies of pharmaceutical care for patients with asthma. Ann Pharmacother. 2005;39(4):625-631.

46. Schommer JC, Pedersen CA, Doucette WR, Gaither CA, Mott DA. Community pharmacists' work activities in the United States during 2000. J Am Pharm Assoc (Wash). 2002;42(3):399-406.

47. Christensen DB, Hansen RW. Characteristics of pharmacies and pharmacists associated with the provision of cognitive services in the community setting. J Am Pharm Assoc (Wash). 1999;39(5):640-649. 


\section{iv) Improvement Studies}

1. Brown WI, Cernusca D, Roehrich L. Evaluation of a Hybrid Training Module for Community Pharmacy Staff Providing Hypertension Medication Therapy Management. J Pharm Pract. 2018;31(2):183-189.

2. Murray ME, Barner JC, Pope ND, Comfort MD. Impact and Feasibility of Implementing a Systematic Approach for Medication Therapy Management in the Community Pharmacy Setting: A Pilot Study. J Pharm Pract. 2018:897190018779847.

3. Mantzourani E, Way CM, Hodson KL. Does an integrated information technology system provide support for community pharmacists undertaking Discharge Medicines Reviews? An exploratory study. Integr Pharm Res Pract. 2017;6:145-156.

4. Jacobs S, Bradley F, Elvey R, et al. Investigating the organisational factors associated with variation in clinical productivity in community pharmacies: a mixed-methods study. Heal Serv Deliv Res. 2017;5(27).

5. Guthrie KD, Stoner SC, Hartwig DM, et al. Physicians' Preferences for Communication of Pharmacist-Provided Medication Therapy Management in Community Pharmacy. J Pharm Pract. 2017;30(1):17-24.

6. Gernant SA, Zillich AJ, Snyder ME. Access to Medical Records' Impact on Community Pharmacist-Delivered Medication Therapy Management: A Pilot From the Medication Safety Research Network of Indiana (Rx-SafeNet). J Pharm Pract. 2017:897190017735422.

7. Greising C, Kossler K, Freyer J, et al. The status of the performance of medication reviews in German community pharmacies and assessment of the practical performance. Int J Clin Pharm. 2016;38(6):1425-1435.

8. Mantzourani E, Ellis L, Parry-Jones G, Hodson K, Way C. Does an integrated information technology system provide support for community pharmacists undertaking discharge medicines reviews? Int J Pharm Pract. 2016;24:82-83.

9. Margolis AR, Martin BA, Mott DA. Trained student pharmacists' telephonic collection of patient medication information: Evaluation of a structured interview tool. J Am Pharm Assoc (2003). 2016;56(2):153-160.

10. Hindi A, Patel N, Rashidi Y, Ho SY, Donyai P. Development and field validation of a new medicines use review patient satisfaction questionnaire based on an existing qualitativelyderived framework. Int J Pharm Pract. 2016;24:47-48. 
11. Pammett RT, Blackburn D, Taylor J, et al. Evaluation of a Community Pharmacy-Based Screening Questionnaire to Identify Patients at Risk for Drug Therapy Problems. Pharmacotherapy. 2015;35(9):881-886.

12. Maes K, Bruch S, Hersberger K, Lampert M. Drug-related problems in community pharmacies: A new tool to classify pharmaceutical interventions. Int J Clin Pharm. 2015;37(1):201.

13. van Lint JA, Sorge LA, Sorensen TD. Access to patients' health records for drug therapy problem determination by pharmacists. J Am Pharm Assoc (2003). 2015:e281-e284.

14. Snyder ME, Pater KS, Frail CK, Hudmon KS, Doebbeling BN, Smith RB. Utility of a brief screening tool for medication-related problems. Res Social Adm Pharm. 2015;11(2):253-264.

15. Mast R, Ahmad A, Hoogenboom SC, et al. Amsterdam tool for clinical medication review: development and testing of a comprehensive tool for pharmacists and general practitioners. BMC Res Notes. 2015;8:642.

16. Hatah E, Tordoff J, Duffull SB, Braund R. Pharmacists' performance of clinical interventions during adherence support medication reviews. Res Social Adm Pharm. 2014;10(1):185-194.

17. Bulloch MN, Olin JL. Instruments for evaluating medication use and prescribing in older adults. J Am Pharm Assoc (2003). 2014;54(5):530-537.

18. Al-Nagar A, Desborough J. A feasibility study to investigate community pharmacy consultations. Int J Pharm Pract. 2014;22:31-32.

19. Morello CM, Hirsch JD, Lee KC. Navigating complex patients using an innovative tool: the MTM Spider Web. J Am Pharm Assoc (2003). 2013;53(5):530-538.

20. Dreischulte T, Johnson J, McAnaw J, Geurts M, de Gier H, Hudson S. Medication assessment tool to detect care issues from routine data: a pilot study in primary care. Int J Clin Pharm. 2013;35(6):1063-1074.

21. Waheedi S, Walker R, Donovan M, John D. An analysis of medicines use review documentation in one community pharmacy. Int J Clin Pharm. 2012;34(1):184.

22. Latif A, Boardman H, Pollock K. Exploring community pharmacy medicines use reviews as a means of improving the clinical and cost effectiveness of prescribed medicines and reducing waste. Pharmacoepidemiol Drug Saf. 2011;20(6):666.

23. Brache E, O'Riordan G, Clemerson J. A peer review of Medicine Use Reviews (MURs) conducted by community pharmacists in Guernsey during the thirteen months after their inception. Int J Pharm Pract. 2011;19:66-67.

24. Kaae S, Sorensen EW, Norgaard LS. Exploring communications around medication review in community pharmacy. Int J Clin Pharm. 2011;33(3):529-536. 
25. MacAdam AB, Sherwood J. An investigation into the provision of Medicines Use Reviews (MURs) by community pharmacists within Brighton and Hove Primary Care Trust. Int J Pharm Pract. 2011;19:31.

26. Trapskin K, Sorum S, Johnson C, Walbr , t Pigarelli D, Decker C. Development of clinical toolkits as support material for Wisconsin Pharmacy Quality Collaborative pharmacists. J Am Pharm Assoc. 2011;51(2):258.

27. Martin BA, Chui MA, Thorpe JM, Mott DA, Kreling DH. Development of a scale to measure pharmacists' self-efficacy in performing medication therapy management services. Res Social Adm Pharm. 2010;6(2):155-161.

28. Bassi M, Wood K. Medicines use reviews: Time for a new name? Int J Pharm Pract. 2009;17:B4-B5.

29. LaFleur J, Larson BS, Gunning KM, et al. Agreement between pharmacists for problem identification: an initial quality measurement of cognitive services. Ann Pharmacother. 2009;43(7):1173-1180.

30. McMahan R. Operationalizing MTM through the use of health information technology. J Manag Care Pharm. 2008;14(2):S18-21.

31. Krska J, Avery AJ, Community Pharmacy Medicines Management Project Evaluation T. Evaluation of medication reviews conducted by community pharmacists: a quantitative analysis of documented issues and recommendations. Br J Clin Pharmacol. 2008;65(3):386-396.

32. James $\mathrm{DH}$, Hatten $\mathrm{S}$, Roberts $\mathrm{D}$, John $\mathrm{DN}$. Identifying criteria for assessing the quality of medicines use review referral documentation by community pharmacists. Int J Pharm Pract. 2008;16(6):365-374.

33. Albers T. Importance of documentation to successful medication therapy management practices. J Am Pharm Assoc (2003). 2006;46(4):426.

34. de Oliveira DR, Shoemaker SJ. Achieving patient centeredness in pharmacy practice: openness and the pharmacist's natural attitude. J Am Pharm Assoc (2003). 2006;46(1):56.

35. McLean WM, MacKeigan LD. When does pharmaceutical care impact health outcomes? A comparison of community pharmacy-based studies of pharmaceutical care for patients with asthma. Ann Pharmacother. 2005;39(4):625-631. 


\section{v) - Methodology \& Practice Tools}

1. Rosenthal MM, Holmes ER. The Professional Culture of Community Pharmacy and the Provision of MTM Services. Pharm (Basel, Switzerland). 2018;6(2).

2. Cardwell K, Hughes CM, Ryan C. Community pharmacists' views of using a screening tool to structure medicines use reviews for older people: findings from qualitative interviews. Int J Clin Pharm. 2018.

3. Hindi A, Patel N, Rashidi Y, Ho SY, Donyai P. Development and field validation of a new medicines use review patient satisfaction questionnaire based on an existing qualitativelyderived framework. Int J Pharm Pract. 2016;24:47-48.

4. Pammett RT, Blackburn D, Taylor J, et al. Evaluation of a Community Pharmacy-Based Screening Questionnaire to Identify Patients at Risk for Drug Therapy Problems. Pharmacotherapy. 2015;35(9):881-886.

5. Maes K, Bruch S, Hersberger K, Lampert M. Drug-related problems in community pharmacies: A new tool to classify pharmaceutical interventions. Int J Clin Pharm. 2015;37(1):201.

6. Snyder ME, Pater KS, Frail CK, Hudmon KS, Doebbeling BN, Smith RB. Utility of a brief screening tool for medication-related problems. Res Social Adm Pharm. 2015;11(2):253-264.

7. Bardet J-D, Vo T-H, Bedouch P, Allenet B. Physicians and community pharmacists collaboration in primary care: A review of specific models. Res Social Adm Pharm. 2015;11(5):602-622.

8. Mast R, Ahmad A, Hoogenboom SC, et al. Amsterdam tool for clinical medication review: development and testing of a comprehensive tool for pharmacists and general practitioners. BMC Res Notes. 2015;8:642.

9. Hasan S, Sulieman H, Stewart K, Chapman CB, Hasan MY, Kong DCM. Assessing patient satisfaction with community pharmacy in the UAE using a newly-validated tool. Res Social Adm Pharm. 2013;9(6):841-850.

10. Kibicho J, Owczarzak J. A patient-centered pharmacy services model of HIV patient care in community pharmacy settings: a theoretical and empirical framework. AIDS Patient Care STDS. 2012;26(1):20-28.

11. Martin BA, Chui MA, Thorpe JM, Mott DA, Kreling DH. Development of a scale to measure pharmacists' self-efficacy in performing medication therapy management services. Res Social Adm Pharm. 2010;6(2):155-161.

12. Pellegrino AN, Martin MT, Tilton JJ, Touchette DR. Medication therapy management services: definitions and outcomes. Drugs. 2009;69(4):393-406. 
13. Academy of Managed Care P. Sound Medication Therapy Management Programs, Version 2.0 with validation study. J Manag Care Pharm. 2008;14(1):S2-44.

14. Wong ICK, Team R. Randomised controlled trials (RCTs) to evaluate complex healthcare interventions--a case study. Pharm World Sci. 2004;26(5):247-252.

15. Roberts AS, Hopp T, Sorensen EW, et al. Understanding practice change in community pharmacy: a qualitative research instrument based on organisational theory. Pharm World Sci. 2003;25(5):227-234.

16. Messerli M, Lottaz V, Vriends N, Hersberger KE. Collaborative development of outcome measures to investigate intermediate medication reviews provided in community pharmacies. Int J Clin Pharm. 35(3):502. 